\title{
Uniform blowing and suction applied to nonuniform adverse-pressure-gradient wing boundary layers
}

\author{
Marco Atzori $\odot^{*}$ and Ricardo Vinuesa \\ SimEx/FLOW, Engineering Mechanics, KTH Royal Institute of Technology, Stockholm 10044, Sweden \\ Alexander Stroh, Davide Gatti $\odot$, and Bettina Frohnapfel \\ Institute of Fluid Mechanics, Karlsruhe of Techonology, Karlsuhe 76131, Germany \\ Philipp Schlatter \\ SimEx/FLOW, Engineering Mechanics, KTH Royal Institute of Technology, Stockholm 10044, Sweden
}

(Received 11 August 2021; accepted 15 November 2021; published 29 November 2021)

\begin{abstract}
A detailed analysis of the effects of uniform blowing, uniform suction, and body-force damping on the turbulent boundary layer developing around a NACA4412 airfoil at moderate Reynolds number is presented. The flow over the suction and the pressure sides of the airfoil is subjected to a nonuniform adverse pressure gradient and a moderate favorable pressure gradient, respectively. We find that the changes in total skin friction due to blowing and suction are not very sensitive to different pressure-gradient conditions or the Reynolds number. However, when blowing and suction are applied to an adverse-pressure-gradient (APG) boundary layer, their impact on properties such as the boundary-layer thickness, the intensity of the wall-normal convection, and turbulent fluctuations are more pronounced. We employ the Fukagata-Iwamoto-Kasagi decomposition [K. Fukagata et al., Phys. Fluids 14, 73 (2002)] and spectral analysis to study the interaction between intense adverse pressure gradient and these control strategies. We find that the control modifies skin-friction contributions differently in adverse-pressure-gradient and zero-pressure-gradient boundary layers. In particular, the control strategies modify considerably both the streamwisedevelopment and the pressure-gradient contributions, which have high magnitude when a strong adverse pressure gradient is present. Blowing and suction also impact the convection of structures in the wall-normal direction. Overall, our results suggest that it is not possible to simply separate pressure-gradient and control effects, a fact to take into account in future studies on control design in practical applications.
\end{abstract}

DOI: 10.1103/PhysRevFluids.6.113904

\section{INTRODUCTION}

The engineering community has studied blowing and suction through porous walls as possible means to modify external flows since the beginning of modern fluid dynamics. Prandtl [1] showed how suction affects separation in the flow around a cylinder, and, later on, he employed this approach to improve airfoil performance in high-lift configurations [2]. On the other hand, blowing was first

\footnotetext{
*atzori@mech.kth.se
}

Published by the American Physical Society under the terms of the Creative Commons Attribution 4.0 International license. Further distribution of this work must maintain attribution to the author(s) and the published article's title, journal citation, and DOI. Funded by Bibsam. 
considered for cooling of surfaces exposed to high-temperature flows [3]. Numerous experiments have been carried out since then, with both technological improvements on how wall transpiration is achieved, and the ambition of reducing the gap between idealized test cases in canonical flows and more complex scenarios with practical applications. The surface of the porous walls employed in early studies [4-6] had a significant influence on the boundary layer, limiting the applicability of this type of control to the cases for which a very strong transpiration intensity was possible. A significant advancement in this field was the development of construction procedures such as that employed for the microblowing technique (MBT) by Hwang [7], which enables the creation of relatively smooth porous surfaces. Subsequently, numerous studies reported the possibility of drag reduction with relatively low blowing intensities [8]. However, a more generalized application of this technology is yet to be achieved, a fact attributed, on the one hand, to implementation difficulties of engineering nature, and, on the other hand, to a lack of knowledge about how this kind of control would perform for complex turbulent flows [9].

These circumstances motivated the most recent experimental campaigns. Kornilov [10] carried out experiments on a Clark-Z airfoil at $\mathrm{Re}_{c}=840000$ (where $\mathrm{Re}_{c}$ is the Reynolds number based on incoming flow velocity $U_{\infty}$, chord length $c$, and fluid viscosity $v$, defined as $\operatorname{Re}_{c}=U_{\infty} c / \nu$ ) and various angles of attack, using a combination of suction at the leading edge and blowing on the pressure side of the airfoil. Subsequently, Eto et al. [11] considered uniform blowing on the suction side of a Clark-Y airfoil at angles of attack from $0^{\circ}$ to $6^{\circ}$ and $\operatorname{Re}_{c}=1500000$, using both active and passive blowing. The results of these two studies confirmed the relevance of test cases as close as possible to realistic scenarios because of the nonobvious effects that this control has on quantities such as total lift and drag. In particular, in both cases, only marginal reduction or even increase of the total drag were achieved. Kornilov et al. [12] employed a combination of uniform blowing on the pressure side and uniform suction on the suction side of the symmetric airfoil NACA0012 at a Reynolds number $\operatorname{Re}_{c}=700000$ and various angles of attack. In the same study, Reynoldsaveraged Navier-Stokes (RANS) simulations were carried out, showing a qualitative agreement with the experiment and, in a later study, an estimate of energy cost for the same setup was provided [13].

High-fidelity numerical simulations have been also employed to study the effects of blowing and suction, but, due to their high computational cost, it has only recently become possible to study external flows at moderately high Reynolds numbers. The first direct numerical simulations (DNS) aimed at evaluating control strategies, including uniform blowing and suction, were carried out using turbulent channel flow as test case $[14,15]$. Note, however, that the most promising application of this type of control is in external flows. Furthermore, in these studies blowing and suction were also used as actuator mechanisms in feedback control, which uses information from sensors to modify the flow interactively, while this study is focused on predetermined control, i.e., with a fixed value of transpiration intensity.

The first DNS of a fully developed turbulent boundary layer (TBL) over a flat plate with uniform blowing and suction, which can be considered a model for a MBT transpirating surface, was performed by Kametani and Fukagata [16] but it was still limited to a very low Reynolds number $\operatorname{Re}_{\theta} \approx 750$. Later on, Kametani et al. [17] performed a similar study with highly resolved large-eddy simulations (LES) extending the Reynolds-number range up to $\operatorname{Re}_{\theta}=2500$, also employing the so-called Fukagata-Iwamoto-Kasagi (FIK) decomposition [18] and spectral analysis to describe in detail how this control modifies the turbulent flow. At the same time, Stroh et al. [19], using opposition control as an example, discussed how control effects vary in turbulent channels and boundary layers. They reported that, even if a similar drag reduction is achieved, the contributions to the skin friction from the FIK decomposition are affected differently in both flows, suggesting that the interactions between control and turbulence may be qualitatively different for internal and external flows. Other studies explored topics such as differences and similarities of uniform blowing and opposition control with similar skin-friction reduction [20], how to better take into account the combined effects of blowing on skin friction, turbulent fluctuations, and boundary-layer thickness [21], and how an asymptotic state could be achieved for a developing turbulent boundary layer under suction $[22,23]$. 
More recently, and similarly to what observed for experiments, high-fidelity simulations have been employed to study cases which are more relevant to practical applications. For instance, Mahfoze et al. [24] discussed the possibility of benefiting from the effects of blowing downstream the control region to optimize the control distribution, and Albers et al. [25] performed a DNS where the effects of a different control strategy (i.e., transversal surface waves) were evaluated on a wing section at $\operatorname{Re}_{c}=400000$.

The lack of detailed simulation data on how blowing and suction may impact APG TBLs motivated the development of the present data set. Following the same methodology as that of a preliminary work carried out at $\mathrm{Re}_{c}=100000$ [26], we performed highly resolved LES with uniform blowing and uniform suction applied on the suction side of a NACA4412 airfoil at $\operatorname{Re}_{c}=200000$ and $\operatorname{Re}_{c}=400000$, as well as a case at $\operatorname{Re}_{c}=200000$ where body-force damping is employed to mimic the effects of opposition control. In previous works, we considered the cases at $\operatorname{Re}_{c}=200000$, focusing on how blowing and suction affect the total drag and lift [27], and we employed Reynolds-averaged Navier-Stokes (RANS) simulations to investigate the possibility of achieving net-energy saving for a larger interval of Reynolds numbers, including an estimate of the actuation cost [28]. In this paper, we focus on the properties of the turbulent boundary layers developing on the wing, to describe how this type of control interacts with the strong adverse pressure gradient. Despite the low to moderate Reynolds number, this study provides interesting information on how intense and nonuniform adverse pressure gradients interact with different control strategies. The choice of the NACA4412 airfoil ensures that the pressure distribution is only moderately dependent on the Reynolds number, allowing comparison with future numerical and experimental studies. The strong pressure-gradient effects remain the most evident feature of this type of flow even at higher $\mathrm{Re}_{c}$, suggesting that our general conclusions also apply for a wider range of conditions.

We organized the material as follows: In Sec. II, we describe the data set; in Sec. III, we discuss how the control affects the streamwise development of the boundary layer, the wall-normal profiles of mean velocity and fluctuations, as well as the budgets of the turbulent kinetic energy from the cases at higher Reynolds number; in Sec. IV, we examine the different contributions to the skin friction, decomposed following the FIK identity; in Sec. V, we present the results of one- and two-dimensional spectral analysis, and in Sec. VI we summarize our results and discuss directions of future development.

\section{METHODOLOGY}

We carried out LES of the incompressible flow around a NACA4412 at the two Reynolds numbers of $\operatorname{Re}_{c}=200000$ and 400000 , considering three control strategies, namely, uniform blowing, uniform suction, and body-force damping, in different configurations.

We employed the spectral-element code Nek5000 [29]. In this code, the computational domain consists of hexahedral elements. Inside each element, Lagrangian interpolants represent velocity and pressure defined along each of the three directions on $N$ points with Gauss-Lobatto-Legendre distribution and $N-2$ points with Gauss-Legendre distribution, respectively. The time integration is performed using a third-order extrapolation scheme for the nonlinear term, and a third-order implicit backward formula for the viscous term. The LES filter is based on the relaxation-term approach described by Schlatter et al. [30], which has an effect similar to that of an implicit filter but preserves continuity and does not depend on the time step. The governing equations of the system that we describe are the incompressible Navier-Stokes momentum equation in typical nondimensional form, including the LES filter that has the physical interpretation of a volume force, and the continuity equation

$$
\frac{\partial u_{i}}{\partial t}+u_{j} \frac{\partial u_{i}}{\partial x_{j}}=-\frac{\partial p}{\partial x_{i}}+\frac{1}{\operatorname{Re}_{c}} \frac{\partial^{2} u_{i}}{\partial x_{j} \partial x_{j}}-\mathcal{H}\left(u_{i}\right), \quad \frac{\partial u_{i}}{\partial x_{i}}=0
$$


In this expression, the summation convention is used for index variables, $u_{i}$ denotes the velocity components in a Cartesian frame of reference, $p$ denotes the pressure, and $\mathcal{H}$ is a high-pass filter, acting on a given subset of modes within each spectral element. A validation of this filter as implemented in Nek5000 was presented by Vinuesa et al. [31].

The grid was designed for both Reynolds numbers so that the spacing in the turbulent region of the domain surrounding the airfoil is $\Delta x_{t}^{+}=18, \Delta y_{n}^{+}=(0.64,11)$, and $\Delta z^{+}=9$ for the wall-tangential, wall-normal, and spanwise directions, respectively. The spacing in inner units is scaled using the viscous length $l^{*}=v / u_{\tau}$. In this expression, the friction velocity is $u_{\tau}=\sqrt{\tau_{w} / \rho}$, and the wall-shear stress at the wall is $\tau_{w}=\rho v\left(d U_{t} / d y_{n}\right)_{y_{n}=0}$, where $\rho$ is the fluid density. The domain extension in the horizontal and vertical directions is $6 c$ and $4 c$, respectively. For cases at $\operatorname{Re}_{c}=200000$, the spanwise width of the computational domain is $0.2 c$, whereas for cases at $\operatorname{Re}_{c}=400000$ the spanwise width is $0.1 c$.

To ensure that the spanwise width of the domain is sufficient to capture the largest turbulent structures, we examined the power-spectral densities of velocity fluctuations. The power-spectral densities are described in Sec. VI, and confirm that the domain width is sufficient to capture the range of active scales in the turbulent boundary layer over the airfoil. We also found that the boundary-layer thickness is always a small fraction of the domain width. In particular, in the region where we examined turbulent statistics, the ratio between $\delta_{99}$ and the domain width is lower than $30 \%$ for every case, as in the study by Albers et al. [25] and the earlier work by Schlatter and Örlü [32]. Note we identified $\delta_{99}$, i.e., the $99 \%$ boundary-layer edge, using the method proposed by Vinuesa et al. [33], based on the concept of the diagnostic scaling [34].

The domain size and the resolution requirements lead to a number of spectral elements of 127000 for $\operatorname{Re}_{c}=200000$, and 268000 for $\operatorname{Re}_{c}=400000$. Since all the simulations are performed using Lagrange polynomials of order 11 for the velocity, these numbers of spectral elements correspond to approximately $220 \times 10^{6}$ and $463 \times 10^{6}$ grid points for the two Reynolds numbers, respectively.

We performed Reynolds-averaged Navier-Stokes (RANS) simulations for the uncontrolled cases at both Reynolds numbers on a much larger computational domain to provide the initial conditions, as well as the Dirichlet conditions for the front, upper, and lower boundaries. The domain of these precursor RANS simulations extends for $200 c$ in any direction from the airfoil surface. The boundary condition for the rear side of the domain is the outlet condition formulated by Dong et al. [35]. We employed the same velocity boundary conditions as in the reference cases for the control cases at the same Reynolds numbers, as discussed by Atzori et al. [27]. For all the cases, transition to turbulence is induced by tripping applied at $x / c=0.1$ on both sides of the airfoil, using a body force with effects similar to the tripping devices in experiments [36]. Note that this methodology was used for the first time on a similar setup by Hosseini et al. [37] (as illustrated in Fig. 1).

Uniform suction and uniform blowing are implemented as a Dirichlet boundary condition on the airfoil surface, defining the horizontal and vertical components of the velocity so that the wallnormal velocity at the wall corresponds to the proper blowing and suction intensity. The bodyforce damping is implemented following the same approach as in [20]. A volume force defined as $g=-\alpha\left(v_{n, x}, v_{n, y}, 0\right)$ is active in the wall-normal region $y_{n}^{+}<20$ over the suction side and in the streamwise region $0.25<x / c<0.86$. Thus, for the case with body-force damping, the momentum equation becomes

$$
\frac{\partial u_{i}}{\partial t}+u_{j} \frac{\partial u_{i}}{\partial x_{j}}=-\frac{\partial p}{\partial x_{i}}+\frac{1}{\operatorname{Re}_{c}} \frac{\partial^{2} u_{i}}{\partial x_{j} \partial x_{j}}-\mathcal{H}\left(u_{i}\right)+g\left(v_{n}\right) .
$$

Note that $v_{n, x}$ and $v_{n, y}$ are the projections on the horizontal and vertical directions, respectively, of the wall-normal component of the instantaneous velocity $v_{n}$ and $\alpha$ is the numerical coefficient that determines the intensity of the body force. We intended to study the effect of body-force damping on the turbulent boundary layer when the skin-friction reduction achieved by the body force is equivalent to that of uniform blowing. However, we observed that it is not possible to 


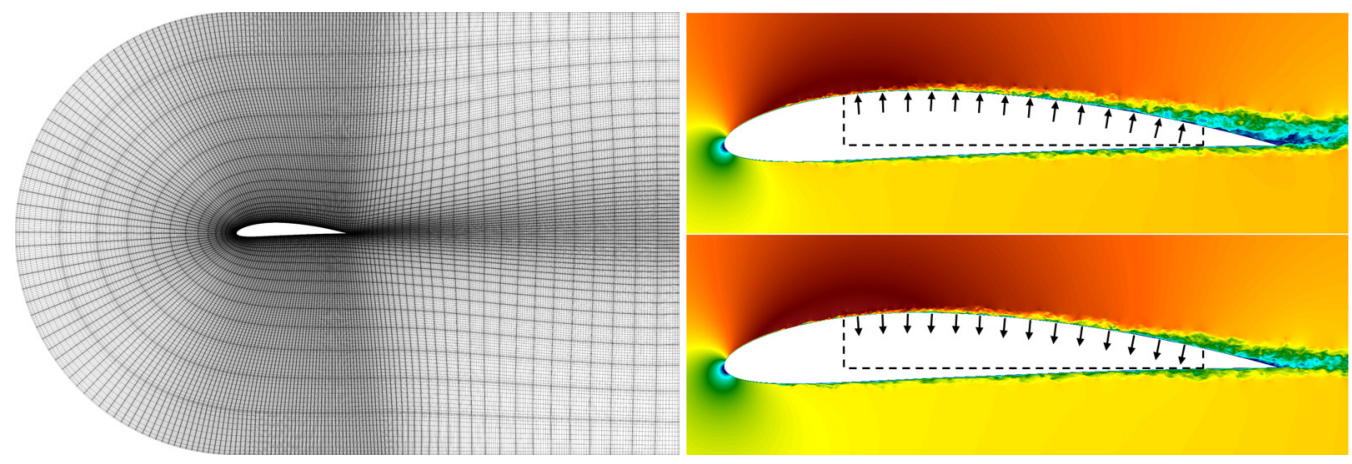

FIG. 1. (Left) Section of the mesh for all the cases at $\mathrm{Re}_{c}=200000$ and horizontal component of the instantaneous velocity in (top right) case $\mathrm{C}$ (intense blowing) and (bottom right) case $\mathrm{E}$ (intense suction) at an arbitrary time step. It is possible to appreciate the different thicknesses of the boundary layer. Velocity values from (blue) $\approx-0.2 U_{\infty}$ to $($ red $) \approx 1.7 U_{\infty}$. Black arrows and lines highlight the control region.

obtain a uniform skin-friction reduction using a uniform value of $\alpha$. To avoid introducing additional complexity, we calibrated $\alpha$ so that the body-force damping results in an integrated skin-friction reduction similar to that of uniform blowing with intensity $0.1 \% U_{\infty}$.

\section{DESCRIPTION OF THE DATA SET}

We consider a total of six different cases with control. In cases BL2 and SC2, we applied uniform blowing and uniform suction, respectively, on the suction side of the airfoil at $\operatorname{Re}_{c}=200000$, between the streamwise locations $x / c=0.25$ and 0.86 . In case $\mathrm{BD} 2$, we applied body-force damping, calibrated to have a similar integrated effect on the skin friction as that of the uniform blowing in case BL2. In case PB2, we applied uniform blowing on the pressure side of the airfoil at $\operatorname{Re}_{c}=200000$, between $x / c=0.2$ and 1.0. Cases BL4 and SC4 denote the same control as that of cases BL2 and SC2, respectively, applied to the airfoil at $\operatorname{Re}_{c}=400000$. The intensity of blowing and suction is $0.1 \% U_{\infty}$ and, for all these cases, the angle of attack is $5^{\circ}$. Note that cases BL2, SC2, and PB2 are part of the data set presented in [27], whereas the reference cases at both Reynolds numbers were previously described in Ref. [31].

The control configurations, as well as their effects on the aerodynamic properties of the airfoil, are summarized in Table I and Fig. 2. Note that the drag and lift coefficients are defined as $C_{d}=$ $f_{d} /(c q)$ and $C_{l}=f_{l} /(c q)$, respectively, where $f_{d} / c$ is the drag force per unit length, $f_{l} / c$ is the lift force per unit length, and $q$ is the dynamic pressure in the free stream $\left(q=\frac{1}{2} \rho U_{\infty}^{2}\right)$. The drag and lift forces per unit lengths are the integrated wall-shear stress and the pressure force projected in the directions parallel and perpendicular to the incoming flow, respectively.

In this case, it is particularly relevant to examine separately the skin-friction drag $C_{d, f}=$ $f_{d, f} /(c q)$, and the pressure drag $C_{d, p}=f_{d, p} /(c q)$, where $f_{d, f}$ and $f_{d, p}$ are the projections in the direction parallel to the incoming flow of the friction and pressure forces, respectively. The values of the integrated lift and drag, as well as that of the skin-friction and pressure contributions to drag, and the aerodynamic efficiency (defined as $C_{l} / C_{d}$ ) are reported in Table I. Uniform blowing reduces $f_{d, f}$ where it is applied, and therefore it reduces $C_{d, f}$, but, if it is applied to the suction side (cases BL2 and BL4), it increases $C_{d, p}$ and eventually results in higher $C_{d}$. To the contrary, uniform suction applied on the suction side increases $C_{d, f}$, but it reduces $C_{d, p}$ and $C_{d}$ (cases SC2 and SC4). Interestingly, both body-force damping and uniform blowing applied on the pressure side reduce both pressure and skin-friction drag. We also performed an exploratory analysis of suction applied on the pressure side, but this configuration reduces lift without having a beneficial effect on drag 
TABLE I. Integrated lift $\left(C_{l}\right)$, integrated skin-friction $\left(C_{d, f}\right)$, and pressure $\left(C_{d, p}\right)$ contributions to the total drag $\left(C_{d}\right)$, and the aerodynamic efficiency $(L / D)$ for the cases considered in this study. The values in parentheses are the relative change with respect to the reference, which is the airfoil at $\operatorname{Re}_{c}=200000$ for cases $\mathrm{BF} 2, \mathrm{SC} 2, \mathrm{BD} 2$, and PB2, and the airfoil at $\mathrm{Re}_{c}=400000$ for Cases BL4 and SC4. The colored squares denote the color code for each case.

\begin{tabular}{|c|c|c|c|c|c|}
\hline Case (notation) & $C_{l}$ & $C_{d, f}$ & $C_{d, p}$ & $C_{d}=C_{d, f}+C_{d, p}$ & $L / D$ \\
\hline \multicolumn{6}{|c|}{$\operatorname{Re}_{c}=200000$} \\
\hline Reference (Ref2 $\square$ ) & 0.867 & 0.0128 & 0.0087 & 0.0215 & 41 \\
\hline Suction side, blowing (BL2 $\square$ ) & $0.833(-4 \%)$ & $0.0122(-4 \%)$ & $0.0099(+14 \%)$ & $0.0221(+3 \%)$ & $38(-7 \%)$ \\
\hline Suction side, suction (SC2 $\square$ ) & $0.898(+4 \%)$ & $0.0133(+4 \%)$ & $0.0076(-12 \%)$ & $0.0210(-2 \%)$ & $43(+6 \%)$ \\
\hline Suction side, body force (BD2 & $0.879(+1 \%)$ & $0.0121(-5 \%)$ & $0.0083(-4 \%)$ & $0.0204(-4 \%)$ & $43(+6 \%)$ \\
\hline Pressure side, blowing (PB2 $\square$ ) & $0.871(+0 \%)$ & $0.0123(-4 \%)$ & $0.0084(-3 \%)$ & $0.0207(-3 \%)$ & $42(+4 \%)$ \\
\hline \multicolumn{6}{|c|}{$\operatorname{Re}_{c}=400000$} \\
\hline Reference (Ref4 $\square$ ) & 0.894 & 0.0109 & 0.0066 & 0.0175 & 51 \\
\hline Suction side, blowing (BL4 $\square$ ) & $0.854(-4 \%)$ & $0.0103(-5 \%)$ & $0.0080(+21 \%)$ & $0.0183(+5 \%)$ & $47(-9 \%)$ \\
\hline Suction side, suction (SC4 & $0.924(+3 \%)$ & $0.0115(+6 \%)$ & $0.0058(-12 \%)$ & $0.0173(-1 \%)$ & $53(+4 \%)$ \\
\hline
\end{tabular}

because the control effect on boundary layer thickness on the pressure side is significantly lower than on the suction side.

A prediction of the actuation cost for these control configurations is difficult to formulate without considering the details of the actuation device, and the strategy adopted to provide air for blowing and to dispose of air for suction. Fahland et al. [28] examined this data set together with results from RANS simulations and formulated an estimate of the energy spent for the control based on

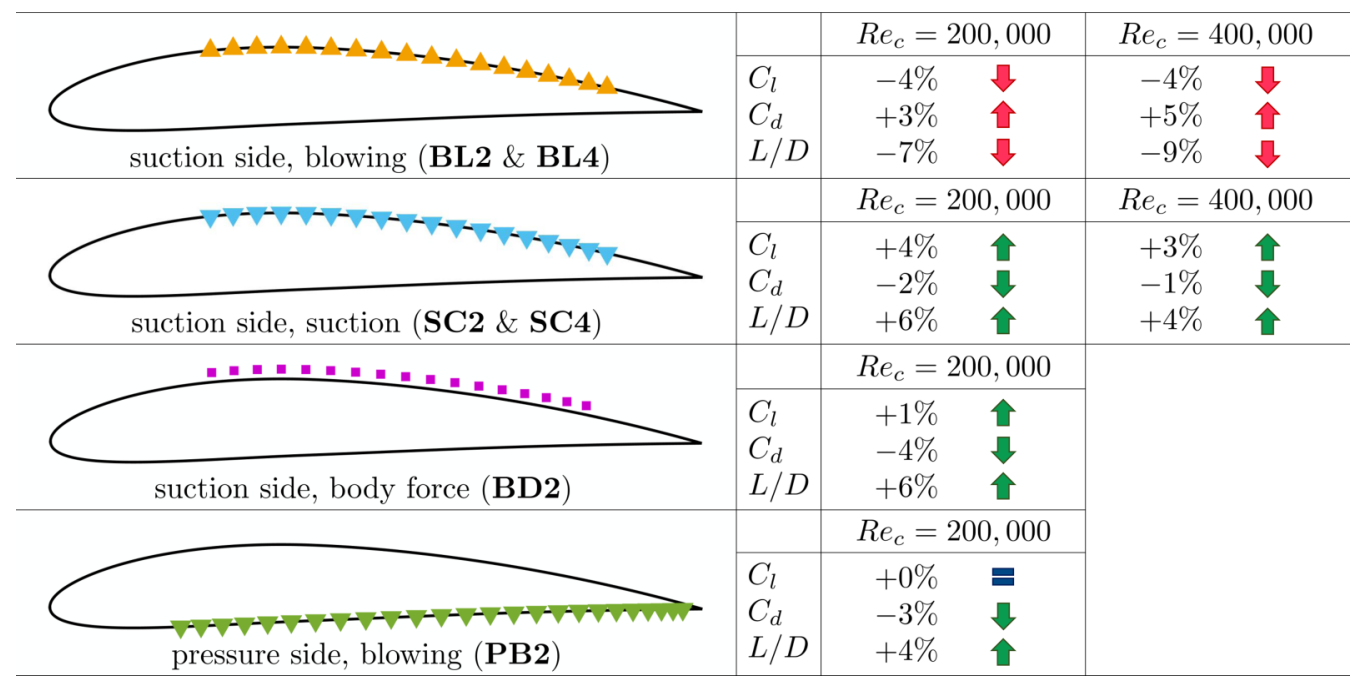

FIG. 2. Summary of the cases considered in this study and relative control effects on the integrated lift $\left(C_{l}\right)$, $\operatorname{drag}\left(C_{d, f}\right)$, and on the aerodynamic efficiency $(L / D)$. Arrows, which point downwards or upwards, denote the increase or decrease of physical quantities, respectively. Green arrows denote control effects that are beneficial for the aerodynamic efficiency (e.g., lift increase or drag reduction), and red arrows denote control effects that are detrimental for the aerodynamic efficiency (e.g., lift reduction or drag increase). A black equal sign indicates only marginal changes. 

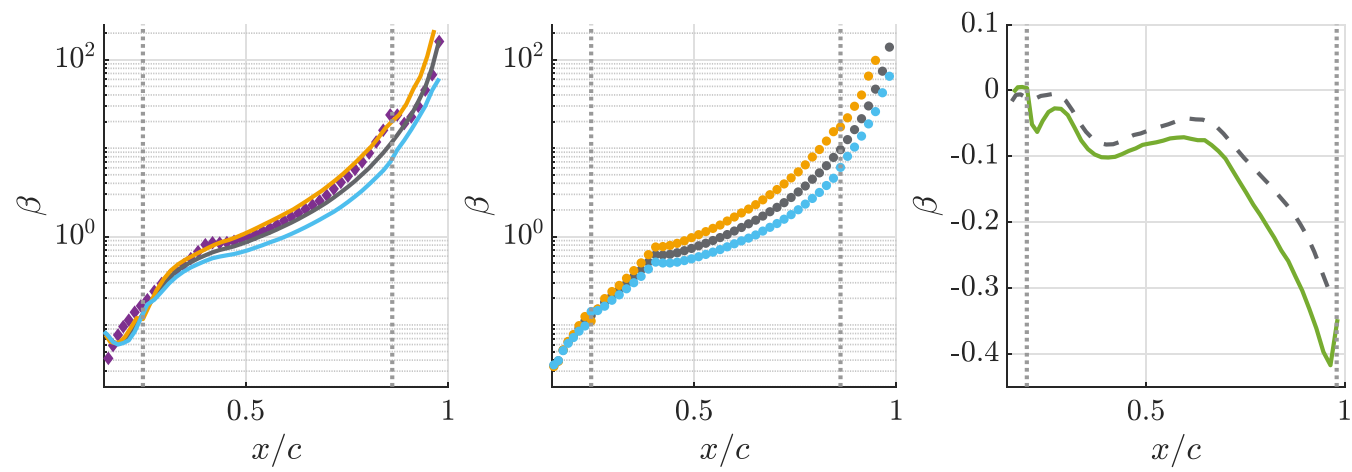

FIG. 3. Clauser pressure-gradient parameter $\beta$ for (left) cases $\mathrm{BL} 2, \mathrm{SC} 2, \mathrm{BD} 2$ and the suction side at $\mathrm{Re}_{c}=200000$, (center) cases BL4 and SC4 and the suction side at $\mathrm{Re}_{c}=400000$, and (right) case PB2 and the pressure side at $\operatorname{Re}_{c}=200000$. The vertical dotted lines indicate the control region. Color code as in Table I.

reasonable pump and turbine efficiencies, finding that blowing applied on the pressure side can give net-energy saving in a wide range of Reynolds numbers.

\section{CONTROL EFFECTS ON FLOW STATISTICS}

In this section, we describe the control effects on the properties of the mean flow for each case, examining both the streamwise development of the boundary layer as well as the wall-normal profiles of the mean velocity components, and the terms of the Reynolds-stress tensor for one streamwise location. In particular, we focus on the comparison between the pressure-gradient and the ZPG cases, and highlight similarities and differences.

\section{A. Streamwise development of the boundary layer}

The turbulent boundary layer developing on the suction side of a NACA4412 airfoil is subjected to a strong adverse pressure gradient, whereas that developing over the pressure side is subjected to a weak favorable pressure gradient. We show the Clauser pressure-gradient parameter $\beta=\delta^{*} / \tau_{w} d P /\left.d x_{t}\right|_{e}$, which is an indicator for the intensity of pressure-gradient effects, in Fig. 3 for all the cases. Note that $\delta^{*}$ and $d P /\left.d x_{t}\right|_{e}$ are the displacement thickness and pressure gradient in the wall-tangential direction at $y_{n}=\delta_{99}$, respectively. On the suction side for both $\operatorname{Re}_{c}, \beta$ exhibits a rapid increase from the location of the maximum chamber to the trailing edge. On the pressure side, $\beta$ is negative, and its absolute value is relatively small because the pressure gradient effects are not very intense.

The control effects on $\beta$ are a consequence of the control effects on $\delta^{*}$ and $\tau_{w}$. Uniform blowing, both on the suction side and the pressure side, reduces $\tau_{w}$, and it increases at the same time $\delta^{*}$, resulting in higher $|\beta|$, and therefore higher $\beta$ on the suction side and lower $\beta$ on the pressure side. On the contrary, uniform suction both increases $\tau_{w}$ and it reduces $\delta^{*}$, resulting in lower $\beta$ on the suction side. Body-force damping has a very small effect on $\delta^{*}$, which is slightly reduced, but it decreases $\tau_{w}$ by a relatively higher magnitude, also resulting in higher $\beta$. The relative variations of boundary-layer thickness and friction velocity determine in a similar way the control effects on other quantities that characterize the boundary-layer development, such as the Reynolds number based on the momentum thickness $\operatorname{Re}_{\theta}=\theta U_{e} / v\left[\theta=\int_{0}^{\delta_{99}} U_{t} / U_{e}\left(1-U_{t} / U_{e}\right) d y\right.$, where $\left.U_{e}=U_{t}\left(\delta_{99}\right)\right]$, and the friction Reynolds number $\operatorname{Re}_{\tau}=\delta_{99} u_{\tau} / \nu$, as illustrated in Fig. 4.

Uniform blowing has effects qualitatively similar on both pressure and suction sides, with one interesting exception. On the suction side, the dominant effect of the control is that on $u_{\tau}$. Therefore, 


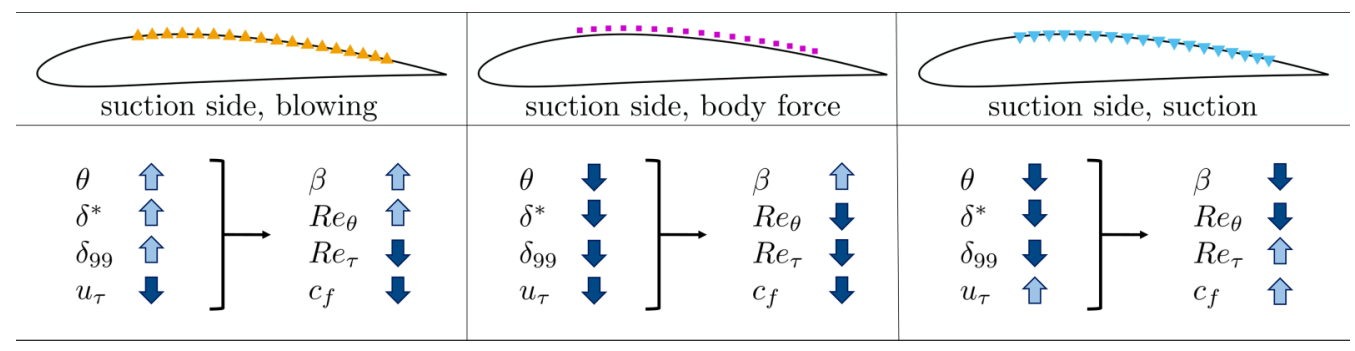

FIG. 4. Summary of the qualitative effects of uniform blowing (cases BL2 and BL4), body-force damping (case BD2), and uniform suction (cases SC2 and SC4) on the development of the APG TBL on the suction side of the airfoil.

uniform blowing in cases BL2 and BL4, which reduces $u_{\tau}$, results in a lower $\operatorname{Re}_{\tau}$ despite the fact that $\delta_{99}$ is higher. On the pressure side, in case PB2, where the streamwise variation of $u_{\tau}$ is less significant, $\operatorname{Re}_{\tau}$ decreases as an effect of uniform blowing only in a small portion of the airfoil, up to $x / c \approx 0.4$, but it increases farther downstream. This fact is due to the increase of $\delta_{99}$, which is proportionally higher than the decrease of $u_{\tau}$.

The control effects on the wall-shear stress discussed above also directly determine the modification of the local skin friction $c_{f}=\tau_{w} /\left(\frac{1}{2} \rho U_{e}^{2}\right)$, which is shown in Fig. 5. As already reported by Stroh et al. [20], the skin-friction reduction (increase) due to uniform blowing (suction) is also present downstream of the control region, although it is lower than where the control is applied. Note that Stroh et al. [20], who examined uniform blowing and body-force damping applied on a zero-pressure gradient (ZPG) boundary layer, described their effects on the turbulent flow downstream the controlled region using the concept of a shifted virtual origin. However, in the present data set, the uncontrolled region is also a region with strong APG, whose local effects depend on the flow history. Because pressure-gradient effects are different for control and uncontrolled cases, the virtual origin argument would require a nontrivial generalization to be applied, taking into account the pressure-gradient distribution.

The comparisons between cases BL2 and BL4, and cases SC2 and SC4 (Fig. 6) show that the effects of blowing and suction on $c_{f}$ have a weak dependence on $\mathrm{Re}_{c}$. In this figure, we compare absolute and relative changes of $c_{f}$ due to control and increase of $\operatorname{Re}_{c}$. Interestingly, $\left|\Delta c_{f}\right|$ for both blowing and suction increases moving downstream, where the adverse pressure gradient is stronger.
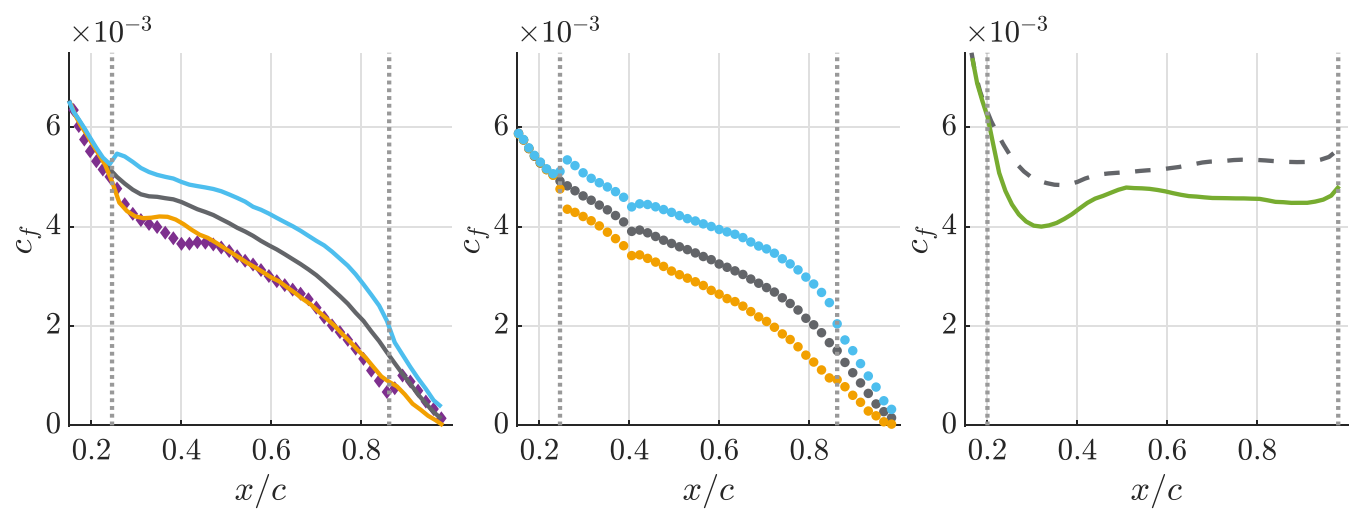

FIG. 5. Local skin-friction coefficient $c_{f}$ for (left) cases BL2, SC2, BD2 and the suction side at $\mathrm{Re}_{c}=$ 200000 , (center) cases BL4 and SC4 and the suction side at $\mathrm{Re}_{c}=400000$, and (right) case PB2 and the pressure side at $\operatorname{Re}_{c}=200000$. The vertical dotted lines indicate the control region. Color code as in Table I. 

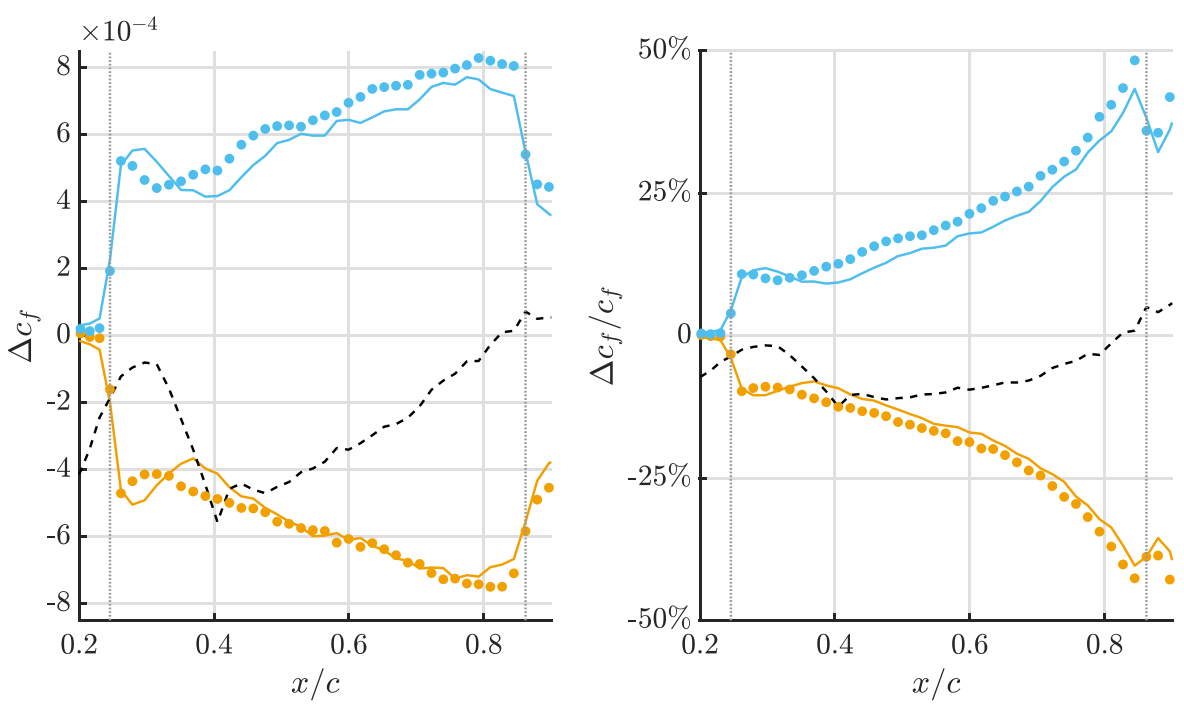

FIG. 6. (Left) Absolute and (right) relative difference in local skin friction for blowing and suction with intensity $0.1 \% U_{\infty}$ at (solid lines) $\operatorname{Re}_{c}=200000$ and (symbols) $\operatorname{Re}_{c}=400000$, and between the uncontrolled cases. Note that the horizontal axis is limited to the control region. The color code is the same as Table I. For each control case, the difference is computed with respect to the uncontrolled case at the same Reynolds number (e.g., the solid blue line is $\Delta c_{f}^{\mathrm{SC} 2}=c_{f}^{\mathrm{SC} 2}-c_{f}^{\mathrm{Ref2}}$ ), and the relative difference is normalized with $c_{f}$ of the uncontrolled case. The black dashed lines indicate the difference between the two uncontrolled cases at $\operatorname{Re}_{c}=400000(\operatorname{Ref} 4)$ and $\operatorname{Re}_{c}=200000$ (Ref2), and the relative difference is normalized with the $c_{f}$ of $\operatorname{Ref} 2$.

Because $c_{f}$ naturally decreases approaching the trailing edge, $\left|\Delta c_{f} / c_{f}\right|$ increases quite significantly, from $\approx 10 \%$ at the beginning of the control region up to more than $\approx 40 \%$ at the end. Furthermore, both $\Delta c_{f}$ and $\Delta c_{f} / c_{f}$ are in relatively good agreement at the two Reynolds numbers considered here.

The skin-friction reduction due to body-force damping (case BD2) is not uniform in the streamwise direction. The value of the numerical parameter $\alpha=32.5$ gives an integrated skin-friction reduction over the suction side of the airfoil that differs from that in case BL2 (blowing with intensity $0.1 \% U_{\infty}$ ) by less than $1 \%$. The local skin-friction reduction is in very good agreement with that of case BL2 only in a certain streamwise region, between approximately $x / c \approx 0.45$ and 0.8 , and it differs elsewhere. On the one hand, it is higher than in case BL2 between $x / c \approx 0.3$ and 0.45 (being as strong as that of uniform blowing with $0.2 \% U_{\infty}$ at $x / c=0.4$ ), and in the control region downstream of $x / c \approx 0.8$, where the adverse pressure gradient is very strong. On the other hand, downstream of the control region, $c_{f}$ in case $\mathrm{BD} 2$ recovers very quickly, being as high as in case $\mathrm{A}$ from $x / c \approx 0.9$ up to the trailing edge.

\section{B. Wall-normal profiles of the mean velocity}

Uniform-blowing and uniform-suction effects on the turbulent statistics of zero-pressure-gradient boundary layers share similarities with those of adverse and favorable pressure gradients, respectively [17]. In previous studies on uniform blowing and suction applied to adverse-pressure-gradient boundary layers, we also observed that there are interactions between this control and pressuregradient effects $[26,27]$. In particular, we found that the mean properties of turbulent boundary layers subjected to adverse pressure gradients are proportionally more affected by blowing and suction than in zero-pressure-gradient boundary layers. 

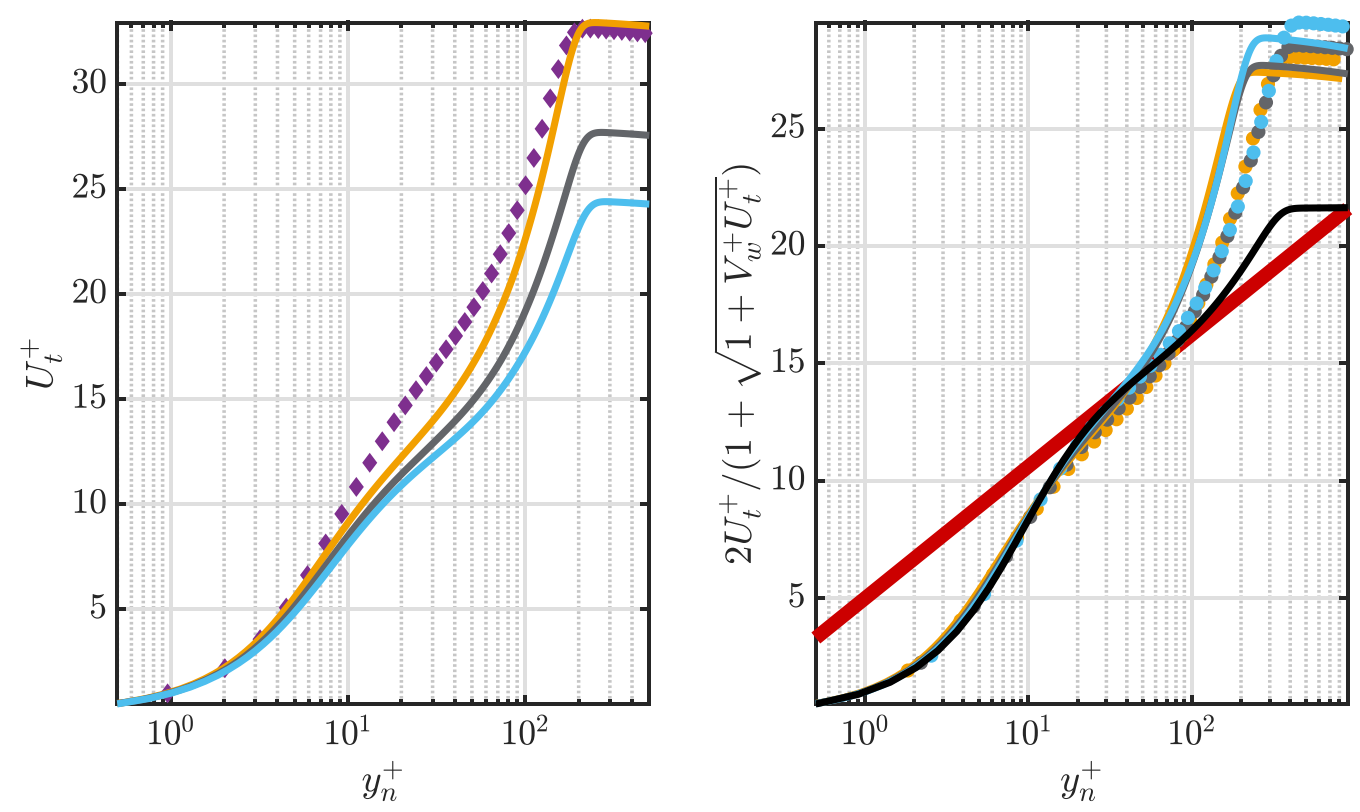

FIG. 7. (Left) Inner-scaled mean wall-tangential velocity component $U_{t}^{+}$as a function of the inner-scaled wall-normal distance $y_{n}^{+}$, and at streamwise location $x / c=0.75$ for cases BL2, SC2, BD2 and the uncontrolled suction side at $\operatorname{Re}_{c}=200000$. Color code as in Table I. (Right) Profiles of the mean velocity scaled following the Stevenson law [39] for the cases with blowing (BL2 and BL4), suction (SC2 and SC4), and the uncontrolled suction sides at both Reynolds numbers. Solid lines for $\operatorname{Re}_{c}=200000$, dots for $\operatorname{Re}_{c}=400000$, and black lines for reference data of ZPG TBL at similar Reynolds number [38]. The red lines in the background are $U_{t}^{+}=1 / K\left(\ln y_{n}^{+}+C\right)$, where $C=2.05$ and $K=0.41$.

We show the inner-scaled wall-normal profiles of the wall-tangential component of the mean velocity in Fig. 7 (left) for all the cases at $\operatorname{Re}_{c}=200000$ at the streamwise location $x / c=0.75$, compared with the same quantity for ZPG boundary layers at similar $\operatorname{Re}_{\tau}$ of the reference case [38]. Note that the inner-scaled mean velocity $U_{t}^{+}$and the wall distance $y_{n}^{+}$are computed using the local wall-shear stress for each case. The profile over the suction side of the airfoil for the reference cases clearly shows the effects of the adverse pressure gradient, which result in a higher velocity in the wake region. Due to the low Reynolds number, the logarithmic region of the boundary layer is virtually absent in this case.

On the suction side, the effects of uniform blowing on the mean tangential velocity are similar to those of an even stronger adverse pressure gradient since uniform blowing causes a higher velocity in the wake region than that of the cases without control. However, uniform blowing also has a small but non-negligible effect on the wall-normal regions closer to the wall. On the pressure side, which has only a low-pressure gradient, the inner-scaled wall-tangential velocity profiles are modified similarly, but with a lower deviation from the reference case (not shown here).

Uniform suction has effects that are qualitatively opposite to those of uniform blowing, thereby resulting in a lower $U_{t}^{+}$through the entire wall-normal profile and, in particular, in the wake region. However, at both Reynolds numbers, the displacement due to uniform suction is always lower than that due to uniform blowing of the same intensity.

The effects of body-force damping (case BD2) on $U_{t}^{+}$are more complex than those of uniform blowing, although the skin-friction reduction is the same in both. In particular, $U_{t}^{+}$is higher then in case BL2 at most wall-normal distances but, at the edge of the boundary layer and farther away is in very good agreement between both cases. 

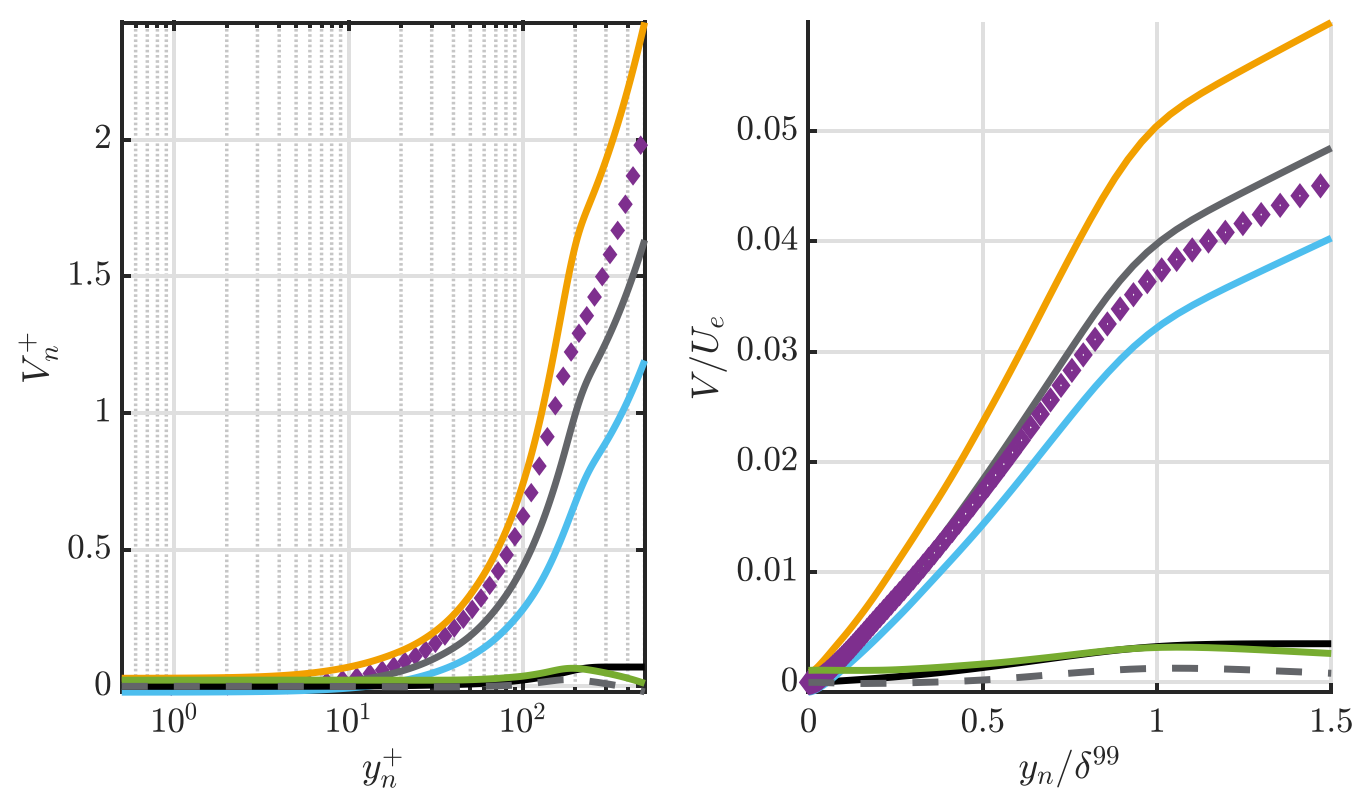

FIG. 8. (Left) Inner-scaled mean wall-normal velocity component $V_{n}^{+}$as a function of the inner-scaled wall distance $y_{n}^{+}$and at $x / c=0.75$, and (right) outer-scaled wall-normal velocity component $V_{n} / U_{e}$ as a function of the outer-scale wall distance $y_{n} / \delta_{99}$ at the same location. Profiles for cases BL2, SC2, BD2, PB2, the uncontrolled cases at $\operatorname{Re}_{c}=200000$, and reference data of ZPG TBL at similar Reynolds number [38]. Color code as in Table I, dashed line for the uncontrolled case on the pressure side (corresponding to case PB2) and black line for ZPG TBL.

In the case of uniform blowing and suction of low intensities, a logarithmic law distinct from the canonical one applies to the wall-tangential velocity, following Stevenson [39]:

$$
\frac{2 U_{t}^{+}}{\sqrt{1+V_{w}^{+} U_{t}^{+}}+1}=\frac{1}{K}\left(\ln y^{+}+C_{0}-C_{1} V_{w}^{+}\right)+O\left(y^{+^{\alpha}}\right) \text {. }
$$

In this expression, $V_{w}^{+}$denotes $V^{+}\left(y_{n}=0\right)$, the inner-scaled value of the wall-normal velocity at the wall, while $K, C_{0}$, and $C_{1}$ are constants. We previously observed that the velocity profiles of the cases with blowing and suction scaled with the Stevenson law collapse on the reference ones despite the strong adverse pressure gradient for the simulations at $\operatorname{Re}_{c}=200000$ [27]. This result also holds for the cases at $\operatorname{Re}_{c}=400000$ (Fig. 7, right), confirming the validity of the Stevenson scaling even though the logarithmic layer is virtually nonexisting. We also tested whether it is possible to obtain a good fit of our data using the composite profile proposed by Nickels [40] for wall-normal profiles of TBL subjected to pressure gradients. This formula is particularly relevant in experimental studies, as it can be used to estimate the local friction coefficient. We obtained a relatively good agreement between the theoretical profiles and the profiles scaled with the Stevenson law (these results are included in the Appendix).

In Fig. 8 (left), we show the wall-normal profiles of the mean velocity component in the wall-normal direction $V_{n}^{+}$for all the cases $\operatorname{Re}_{c}=200000$ at $x / c=0.75$. The strong wall-normal convection is characteristic of APG boundary layers, as it appears comparing the uncontrolled case with the ZPG reference, and uniform blowing and uniform suction directly affect it. In particular, $V_{n}^{+}$ is higher at any wall normal for uniform blowing (case BL2), and lower for uniform suction (case $\mathrm{SC} 2$ ). In the near-wall region $\left(y_{n}^{+}<10\right), V_{n}^{+}$has the same value which is prescribed at the wall and, farther from the wall, it gradually increases following a trend similar to that of the uncontrolled case. On the pressure side, $V_{n}^{+}$in the uncontrolled case is lower than in the ZPG reference for 

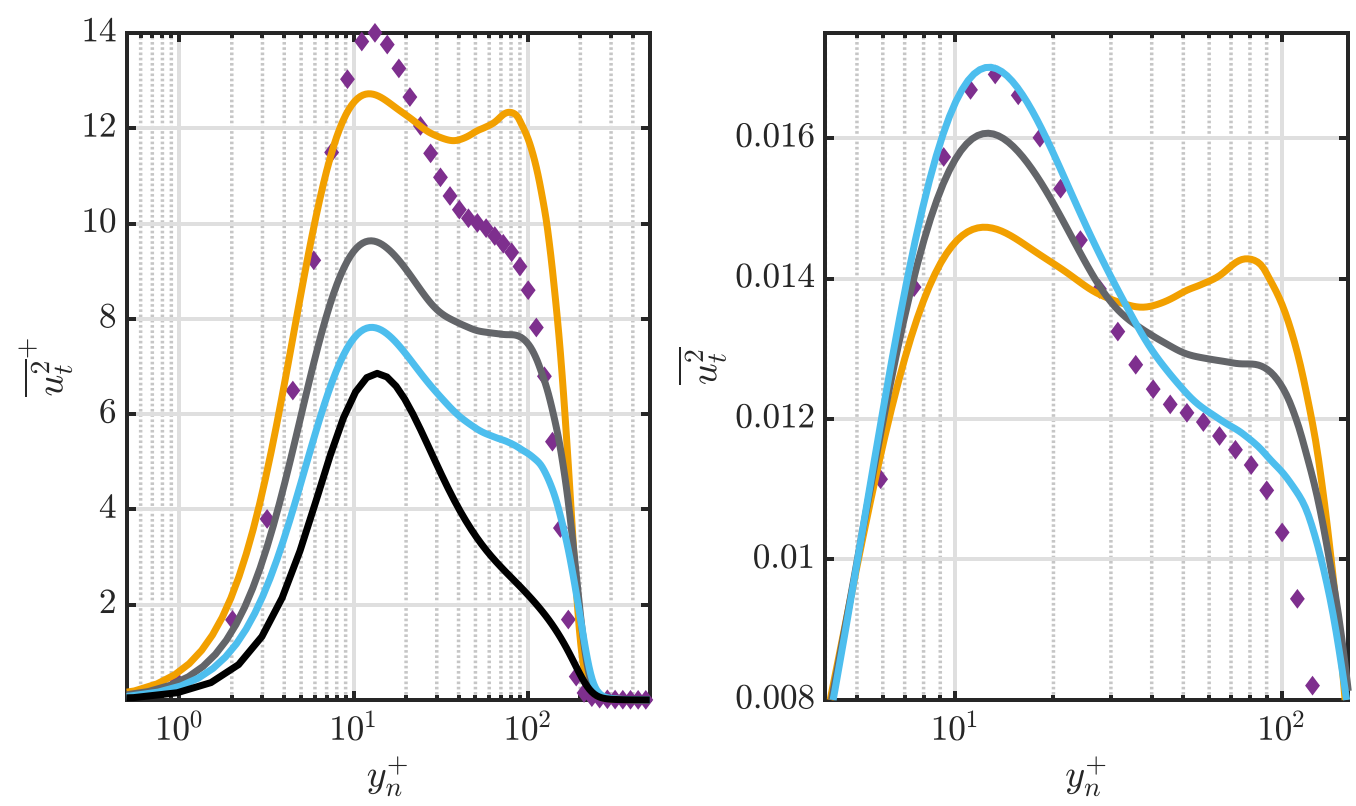

FIG. 9. (Left) Inner- and (right) outer-scaled fluctuations of wall-tangential velocity components $\overline{u_{t}^{2}}$ and $\overline{u_{t} v_{n}}$ as functions of the inner-scaled wall-normal distance $y_{n}^{+}$, for cases BL2, SC2, BD2 and the uncontrolled suction side at $\operatorname{Re}_{c}=200000$ at location $x / c=0.75$. Color code as in Table I, black lines for reference data of ZPG TBL at similar friction Reynolds number [38].

$y_{n}^{+}>10$, because of the mild favorable pressure gradient, and it eventually becomes negative in the wake region. Uniform blowing on the pressure side (case PB2) also results in higher $V_{n}^{+}$, but its values remain much closer to those of the uncontrolled cases than over the suction side. The body-force damping (case BD2) does not affect $V_{n}^{+}$as dramatically as $U_{t}^{+}$. To the contrary, the wall-normal convection follows the same trend as for the other cases, and $V_{n}^{+}$exhibits intermediate values between that of the uncontrolled case and uniform blowing (case BL2), although $\tau_{w}$ cases $\mathrm{BL} 2$ and BD2 are the same.

Increase or decrease of the inner-scaled wall-normal profile can result from a change of friction velocity. We also examined the outer-scaled profiles, which are shown in Fig. 8 (right). The outerscaled wall-normal convection in case BD2 is slightly lower than that of the reference case, despite the fact that the inner-scaled values are higher, meaning that body-force damping does not increase wall-normal convection directly. To the contrary, the outer-scaled profiles for blowing (BL2 and PS2) and suction (SC2) are higher and lower, respectively, than those of the uncontrolled case. On the pressure side, uniform blowing results in an almost constant increase of $V$ at all wall-normal distances. On the other hand, on the suction side, blowing results not only in a higher increase of $V$ than on the pressure side, but such an increase is more prominent farther from the wall. Similarly, the reduction of $V$ due to suction is more severe in the outer region, where the mean wall-normal convection is higher.

\section{Wall-normal profiles of the Reynolds-stress components}

The strong adverse pressure gradient on the suction side of the airfoil has noticeable effects on the distributions of turbulent fluctuations and therefore on the wall-normal profiles of the terms of the Reynolds-stress tensor. In Fig. 9, we illustrate the inner- and outer-scaled profiles of the wall-tangential fluctuations ${\overline{u_{t}^{2}}}^{+}$and $\overline{u_{t}^{2}}$, respectively. 
Note that the comparison between inner- and outer-scaled profiles is relevant in the present context to better distinguish differences between the effects of variations of the friction velocity and modification of the profile shapes.

On the suction side, the comparison between the uncontrolled cases and the ZPG reference shows that the inner-scaled wall-tangential fluctuations are higher at any wall-normal distance. This fact is due to the friction velocity being lower for the APG turbulent boundary layer. Furthermore, an outer peak of ${\overline{u_{t}^{2}}}^{+}$appears, at $y^{+} \approx 75$ in the uncontrolled case, which is not present in the ZPG. Similarly to a stronger APG, uniform blowing gives even higher ${\overline{u_{t}^{2}}}^{+}$because of the lower friction velocity, and a more prominent outer peak, while uniform suction has the opposite effect. Examining the outer-scaled profiles, however, unveils that the inner peak of wall-tangential fluctuations is less intense in the APG uncontrolled cases than in the ZPG references [41], and even lower in cases with uniform blowing.

On the pressure side, the discrepancies between the uncontrolled case and the ZPG reference are small because of the small pressure gradient, and blowing effects are also less significant. As on the suction side, uniform blowing results in higher ${\overline{u_{t}^{2}}}^{+}$at all wall-normal distances, but the outer-scaled profiles of the uncontrolled and controlled cases remain quite similar (not shown). The effects of body-force damping (case BD2) on $\bar{u}_{t}^{+}$and $\overline{u_{t}^{2}}$ are qualitatively different than those of uniform blowing and suction. On the one hand, the inner-scaled profile has similar values to that of uniform blowing with $0.1 \% U_{\infty}$ intensity (case BL2) because $u_{\tau}$ is the same in both cases, but the shape of the profile is different, and the inner peak is higher than the outer peak. On the other hand, in the outer-scaled profile, the inner peak is higher than in the uncontrolled case, and the outer peak is lower than in the reference, so that profile is in good agreement with that of uniform suction with $0.1 \% U_{\infty}$ intensity (case SC2).

The modification of the wall-normal profiles of the Reynolds shear stress, $\overline{u_{t} v_{n}}+$ and $\overline{u_{t} v_{n}}$, caused by pressure gradients and uniform blowing and suction are simpler to describe than that on the wall-tangential fluctuations (the profiles are not shown). Due to the adverse pressure gradient, the location of maximum intensity of the shear stress moves farther away from the wall than in the ZPG reference, to a location roughly corresponding to that of the outer peak of $\overline{u_{t} u_{t}}$. Furthermore, the absolute values of both $\overline{u_{t} v_{n}}+$ and $\overline{u_{t} v_{n}}$ are higher than in ZPG. Uniform blowing and uniform suction lead to more and less intense shear stress, respectively, which is equivalent to an even stronger adverse pressure gradient for uniform blowing and a favorable pressure gradient for uniform suction. Body-force-damping effects again qualitatively differ from those of uniform blowing and suction. In particular, $\left|\overline{u_{t} v_{n}}\right|^{+}$is higher than in the reference up to $y_{n}^{+} \approx 100$, a fact this is due to the lower value of $u_{\tau}$, but $\left|\overline{u_{t} v_{n}}\right|$ is always lower than the reference at any wall-normal distance. The pressuregradient and control effects on the vertical and the spanwise velocity fluctuations $\bar{v}_{n}^{+}$and ${\overline{w^{2}}}^{+}$are similar to those on the Reynolds shear stress $\overline{u v}$ (the profiles are not shown). The control effects on the turbulent statistics are qualitatively illustrated in Fig. 10.

\section{CONTRIBUTIONS TO THE SKIN FRICTION}

\section{A. Reference cases}

With the aim of better describing how the force balance within the TBL is affected by the various control strategies, we examined the different contributions to the skin friction according to the so-called FIK identity [18]. The choice of using the FIK decomposition in the present context rather than, e.g., that based on the vorticity equation proposed by Yoon et al. [42] or the one derived from the budget of the turbulent-kinetic-energy budget proposed by Renard and Deck [43], is mainly motivated by the ease of comparing with previous results in the literature, which are more abundant for the FIK. The decomposition proposed in Ref. [42] follows the same integration procedure than the FIK, and thus there is no reason to consider it more effective a priori. The decomposition proposed in Ref. [43] is derived following a more straightforward approach, and its terms are 


\begin{tabular}{|c|c|c|c|c|c|}
\hline & $U_{t}^{+}, V_{n}^{+}$ & ${\overline{u_{t}^{2}}}^{+},{\overline{u_{t} v_{n}}}^{+}$ & $\overline{u_{t}^{2}}\left(y^{+}<30\right)$ & $\overline{\overline{u_{t}^{2}}}\left(y^{+}>30\right)$ & $\overline{u_{t} v_{n}}$ \\
\hline suction side $(\mathrm{APG})$ & 仓仓 & 仓仓 & $\nabla v$ & 仓仓 & 仓仓 \\
\hline suction side, blowing & 仓仓 & 仓仓 & $\nabla \nabla$ & نे & 仓仓 \\
\hline pressure side, blowing & 仓 & 仓 & $\nabla$ & 仓 & 仓 \\
\hline suction side, suction & 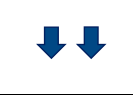 & $\checkmark v$ & 仓仓 & $\nabla$ & $\nabla$ \\
\hline suction side, body force & 仓仓 & 仓仓 & 仓仓 & $\nabla v$ & $\nabla$ \\
\hline
\end{tabular}

FIG. 10. Summary of the qualitative APG and control effects on the turbulent statistics relative to ZPG TBL. Light arrows that point downwards and dark arrows that point upwards denote the increase or decrease of the physical quantity, respectively. Repeated arrows indicate stronger control effects in relative terms.

arguably more easily interpreted than those of the FIK decomposition. However, we compared the results with the ones obtained with the FIK decomposition, we grouped together similar terms, and found the same qualitative behavior (the comparison is reported in the Appendix). The conclusions presented in this section are thus not affected by the choice of the skin-friction decomposition, and provide a general qualitative indication on how the skin-friction contributions are altered by the combined pressure-gradient and control effects.

In the present case, the FIK identity is obtained by integrating by parts the momentum equation on the wall-normal profiles up to $y_{n}=\delta_{99}$. The different terms are simplified by repeating integration by parts and are typically organized into four contributions, denoted as follows:

$$
c_{f}\left(x_{t}\right)=c_{f}^{\delta}\left(x_{t}\right)+c_{f}^{T}\left(x_{t}\right)+c_{f}^{D}\left(x_{t}\right)+c_{f}^{P}\left(x_{t}\right) .
$$

We refer to [43] for a complete derivation in case of boundary layers subjected to pressure gradient. The first contribution $c_{f}^{\delta}$ is directly related to the boundary-layer thickness, and it reads as

$$
c_{f}^{\delta}=\frac{4\left(1-\delta^{*} / \delta_{99}\right)}{\operatorname{Re}_{\delta}} .
$$

The second contribution $c_{f}^{T}$ can be written as

$$
c_{f}^{T}=\int_{0}^{1} \xi_{f}^{T}(\eta) d \eta, \quad \text { where } \quad \xi_{f}^{T}(\eta)=-4(1-\eta) \frac{\overline{u_{t} v_{n}}}{U_{e}^{2}} .
$$

Note that the integration variable is the wall-normal distance normalized with the boundary-layer thickness, i.e., $\eta=y_{n} / \delta_{99}$. The third contribution $c_{f}^{D}$ reads as

$$
c_{f}^{D}=-2 \int_{0}^{1}(1-\eta)^{2} \frac{\delta_{99}}{U_{e}^{2}} I_{x} d \eta
$$

where

$$
I_{x}=\frac{\partial\left(U_{t} U_{t}\right)}{\partial x_{t}}+\frac{\partial^{2} \overline{u_{t} u_{t}}}{\partial x_{t}^{2}}+\frac{\partial\left(U_{t} V_{n}\right)}{\partial \eta}-\frac{1}{\operatorname{Re}_{\delta}} \frac{\partial^{2} U_{t}}{\partial x_{t}^{2}} .
$$

The symbol $I_{x}$ includes all the terms of the momentum equation that are zero if the flow is homogeneous in the wall-tangential direction, so that this contribution accounts for the boundary-layer 


\begin{tabular}{|c|c|c|c|c|c|c|c|}
\hline \multirow{2}{*}{$x / c=0.75$} & \multirow{2}{*}{$c_{f}^{\delta} / c_{f}$} & \multirow{2}{*}{$c_{f}^{T} / c_{f}$} & $c_{f}^{D 1} / c_{f}$ & $c_{f}^{D 2} / c_{f}$ & $c_{f}^{D 3} / c_{f}$ & $c_{f}^{D 4} / c_{f}$ & \multirow{2}{*}{$c_{f}^{P} / c_{f}$} \\
\hline & & & \multicolumn{4}{|c|}{$c_{f}^{D} / c_{f}$} & \\
\hline & \multirow{2}{*}{$17 \%$} & \multirow{2}{*}{$178 \%$} & $1025 \%$ & $14 \%$ & $-675 \%$ & $-3 \%$ & \multirow{2}{*}{$-456 \%$} \\
\hline suction side (APG & & & \multicolumn{4}{|c|}{$361 \%$} & \\
\hline$R e_{c}=200,000$ & \multirow{2}{*}{$18 \%$} & \multirow{2}{*}{$59 \%$} & $9 \%$ & $0 \%$ & $-4 \%$ & $0 \%$ & \multirow{2}{*}{$18 \%$} \\
\hline pressure side (ZPC & & & \multicolumn{4}{|c|}{$5 \%$} & \\
\hline$R e_{c}=400,000$ & \multirow{2}{*}{$11 \%$} & \multirow{2}{*}{$166 \%$} & $924 \%$ & $6 \%$ & $-597 \%$ & $-3 \%$ & \multirow{2}{*}{$-408 \%$} \\
\hline suction side (APG & & & \multicolumn{4}{|c|}{$331 \%$} & \\
\hline
\end{tabular}

FIG. 11. Relative contributions of the terms of the FIK decomposition to the total $c_{f}$ in the uncontrolled cases. By definition, $c_{f}^{D}=c_{f}^{D 1}+c_{f}^{D 2}+c_{f}^{D 3}+c_{f}^{D 4}$ and $c_{f}^{\delta} / c_{f}+c_{f}^{T} / c_{f}+c_{f}^{D} / c_{f}+c_{f}^{P} / c_{f}=100 \%$. Positive and negative percentages denote positive and negative contributions, respectively. Colored cells denote the most relevant contributions (darker colors for higher values).

development. We can have a better understanding of pressure-gradient and control effects on $c_{f}^{D}$ by considering separately the four integrals of the terms included in $I_{x}$, denoted by $c_{f}^{D 1}, c_{f}^{D 2}, c_{f}^{D 3}$, and $c_{f}^{D 4}$ (by definition, $c_{f}^{D}=c_{f}^{D 1}+c_{f}^{D 2}+c_{f}^{D 3}+c_{f}^{D 4}$ ). We observed that the second term, because of the relatively low values of the second derivative of the wall-tangential fluctuations, and the fourth term, which is proportional to the inverse of $\mathrm{Re}_{\delta}$, are virtually negligible for all the cases. The first and third terms take into account streamwise development of the wall-tangential velocity and wall-normal convection, respectively, and can be written as

$$
c_{f}^{D 1}=\int_{0}^{1} \xi_{f}^{D 1}(\eta) d \eta, \quad \text { where } \quad \xi_{f}^{D 1}(\eta)=-4(1-\eta)^{2} \frac{\delta_{99}}{U_{e}^{2}} U_{t} \frac{\partial U_{t}}{\partial x_{t}}
$$

and

$$
c_{f}^{D 3}=\int_{0}^{1} \xi_{f}^{D 3}(\eta) d \eta, \quad \text { where } \quad \xi_{f}^{D 3}(\eta)=-2(1-\eta) \frac{U_{t} V_{n}}{U_{e}^{2}} .
$$

The last contribution $c_{f}^{P}$ includes the pressure gradient explicitly:

$$
c_{f}^{P}=\int_{0}^{1} \xi_{f}^{P}(\eta) d \eta, \quad \text { where } \quad \xi_{f}^{P}(\eta)=-2(1-\eta)^{2} \frac{\delta_{99}}{U_{e}^{2}} \frac{\partial P}{\partial x_{t}} .
$$

We first discuss the effects of the strong adverse pressure gradient present on the suction side, considering relative contributions to the local $c_{f}$ for the reference cases at $x / c=0.75$ (Fig. 11). Note that the relative contributions are defined as values of the contributions divided by $c_{f}$, such that their sum adds up to $100 \%$.

In the present cases, $c_{f}^{\delta}$ is small but not negligible due to the fact that the Reynolds number is relatively low and, at $\operatorname{Re}_{c}=200000$ it accounts for $17 \%$ and $18 \%$ of $c_{f}$ on suction and pressure sides, respectively. As expected, it decreases for larger $x / c$, and it is lower for the cases at $\operatorname{Re}_{c}=$ 400000.

The $c_{f}^{T}$ contribution is the only one where turbulent fluctuations are integrated directly together with the term $(1-\eta)$, and its order of magnitude is comparable with that of the total $c_{f}$. In general, this term is relatively small at low $x / c$, and it increases downstream, as the turbulent boundary layer develops. On the suction side, this contribution increases monotonically even though the total $c_{f}$ decreases monotonically. This is due to the more intense turbulent fluctuations in the outer layer 
caused by the strong adverse pressure gradient. On the other hand, on the pressure side, $c_{f}^{T}$ increases only up to a certain location, which is $x / c \approx 0.65$ for the uncontrolled case, it remains almost stationary farther downstream, and it eventually decreases because of the favorable pressure gradient in the proximity of the trailing edge. On this side, $c_{f}^{T}$ is the most relevant contribution.

The $c_{f}^{D}$ contribution is very relevant on the suction side, where the adverse pressure gradient is progressively stronger towards the trailing edge. In particular, it is relatively small at low $x / c$ but increases significantly downstream, eventually becoming as high as several times the total $c_{f}$. To the contrary, on the pressure side, $c_{f}^{D}$ is much lower than $c_{f}$, reaching only $5 \% c_{f}$. Note that the increase of $c_{f}^{D}$ on the suction side, although it is due to the adverse pressure gradient, is opposite to the pressure-gradient effects on the total $c_{f}$, which decreases. The importance of the $c_{f}^{D}$ contribution is due to the fact that the integrands of $c_{f}^{D 1}$ and $c_{f}^{D 3}$ contain the mean velocity explicitly, so they both have very high absolute values in the region with strong APG. The $c_{f}^{D 1}$ term is positive because $d U_{t} / d x_{t}$ is negative and it has a behavior as a function of $x / c$ similar to that of $c_{f}^{D}$, quickly increasing as the APG becomes stronger. However, $c_{f}^{D 1}$ is even higher than $c_{f}^{D}$, being more than 10 times larger than the total $c_{f}$ where the adverse pressure gradient is particularly strong for the case at $\operatorname{Re}_{c}=$ 200000 . On the other hand, $c_{f}^{D 3}$ exhibits high absolute values but it is negative, thus it balances $c_{f}^{D 1}$. On the pressure side, where a moderate favorable pressure is present, $c_{f}^{D 1}$ and $c_{f}^{D 3}$ are both much less intense than on the suction side, being of the same order as the total $c_{f}$, and $c_{f}^{D 1}$ decreases moving downstream.

The pressure-gradient term $c_{f}^{P}$ gives a negative contribution to the skin friction on the suction side, and a positive contribution on the pressure side. Note that $d P / d x_{t}$ is positive at any wall-normal distance on the suction side, and negative on the pressure side, in regions with a non-negligible favorable pressure gradient. Interestingly, this term is also the only one which decreases as the adverse pressure gradient becomes stronger, while both $c_{f}^{T}$ and $c_{f}^{D}$ increase, and it is therefore responsible for the total $c_{f}$ being lower for an adverse-pressure-gradient boundary layer. To the contrary, $c_{f}^{P}$ increases in the region of the pressure side where the favorable pressure gradient is more intense but such an increase balances the decrease of $c_{f}^{D}$ at the same locations so that the total $c_{f}$ is approximately uniform.

\section{B. Interpretation of FIK contributions in APG TBL}

It is possible to draw a relation between the two terms including the pressure gradient $c_{f}^{P}$ and the streamwise derivative of mean wall-tangential velocity $c_{f}^{D 1}$ if the latter is interpreted as a contribution from the dynamic pressure. Assuming that the Bernoulli equation holds, we can write

$$
\frac{1}{2} U^{2}+p \approx \text { constant } \Rightarrow \frac{1}{2} \frac{\partial}{\partial x}\left(U^{2}\right) \approx-\frac{\partial P}{\partial x},
$$

which would be an exact equality in the case of irrotational flow along streamlines. Using this approximate expression in Eq. (9), it is possible to write

$$
\xi_{f}^{D 1} \approx+4\left(1-\eta^{2}\right) \frac{\delta_{99}}{U_{e}^{2}} \frac{\partial P}{\partial x_{t}} \approx-2 \xi_{f}^{P} \Rightarrow c_{f}^{D 1} \approx-2 c_{f}^{P} .
$$

This relation is, in fact, relatively close to the results reported in Fig. 11, suggesting that these terms are indeed related to each other. The good agreement between $c_{f}^{D 1}$ and $-2 c_{f}^{P}$ is explained by the fact that the Bernoulli equation is fulfilled in the free stream and $d P / d y$ is relatively small by virtue of the boundary-layer approximation, so that the free-stream velocity still has an imprint even within the rotational boundary layer. This effect is also supported by the functional shape of the multiplicative prefactor $\left(1-\eta^{2}\right)$, which gives higher weight to the region far from the wall.

A first implication of the connection between $c_{f}^{D 1}$ and $c_{f}^{P}$ is that these two terms can be considered together in the friction decomposition. Neglecting $c_{f}^{\delta}, c_{f}^{D 2}$, and $c_{f}^{D 4}$, which are one order 

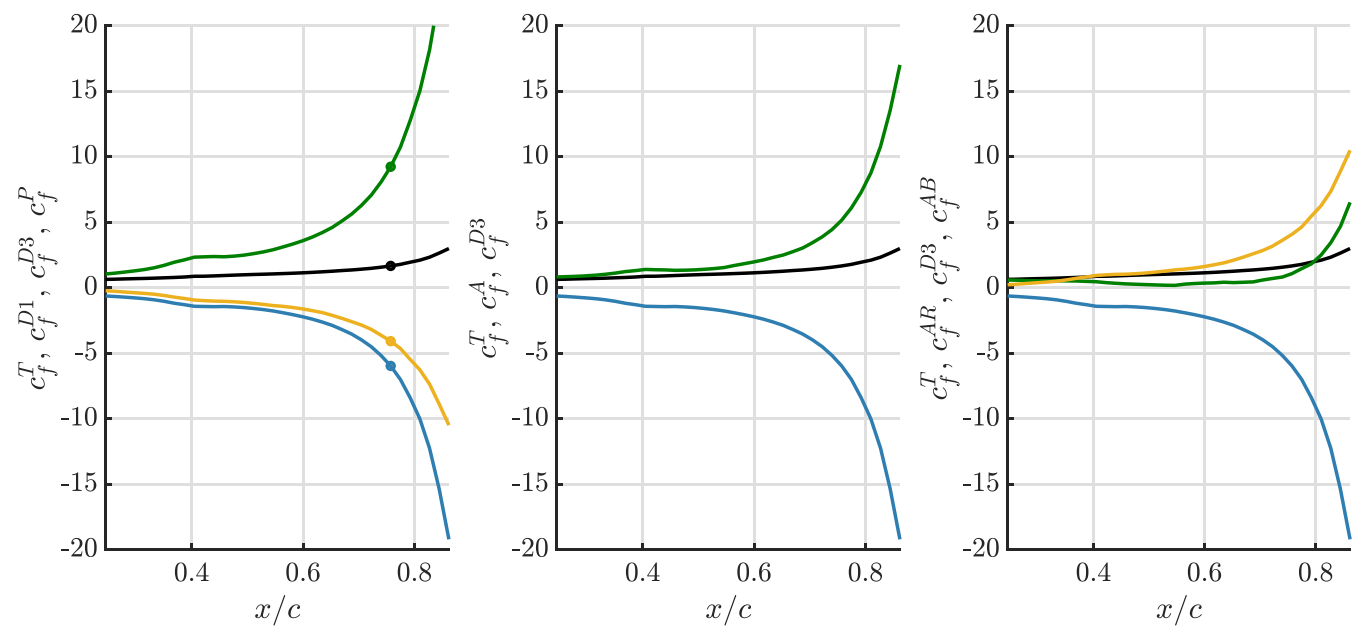

FIG. 12. Streamwise development of the largest relative contributions to friction for the suction side of the reference case at $\operatorname{Re}_{c}=400000$, (left) using the standard FIK formulation [Eq. (14)], (center) introducing the acceleration contribution [Eq. (15)], and (right) introducing the rotational-acceleration and Bernoulli contributions [Eq. (17)]. The relative contributions are normalized with the total $c_{f}$ and their sum is $\approx 0.9$ because $c_{f}^{\delta}, c_{f}^{D 2}$, and $c_{f}^{D 4}$ are neglected. The turbulent-fluctuation contribution, denoted by $c_{f}^{T}$ ( $\square$ ), and the wallnormal-convection contribution, denoted by $c_{f}^{D 1}(\square)$, are the same in all panels. The streamwise-development and the pressure-gradient contributions in the left panel are denoted by $c_{f}^{D 3}(\square)$ and $c_{f}^{P}(\square)$, respectively. The acceleration contribution in the center panel is denoted by $c_{f}^{A}(\square)$. The rotational acceleration and the Bernoulli contributions in the right panel are denoted by $c_{f}^{A R}(\square)$ and $c_{f}^{A B}(\square)$, respectively. Note that $c_{f}^{A B}=-c_{f}^{P}$. The symbols in the left panel indicate the values at $x / c=0.75$, which are reported in Fig. 13.

of magnitude lower than the other contributions, the FIK identity in its traditional form can be written as

$$
c_{f}=c_{f}^{T}+c_{f}^{D 1}+c_{f}^{D 3}+c_{f}^{P} .
$$

We can introduce an acceleration contribution, denoted $c_{f}^{A}$, defined as the sum of $c_{f}^{D 1}$ and $c_{f}^{P}$. In the case of adverse-pressure-gradient boundary layers this term is, in fact, related to the deceleration of the flow and it has a positive sign, as shown in Fig. 12 (center). The FIK identity can thus be rewritten as

$$
c_{f}=c_{f}^{T}+c_{f}^{A}+c_{f}^{D 3} .
$$

The introduction of the acceleration term $c_{f}^{A}$ allows to simplify the description of pressuregradient effects on the skin friction. The lower skin friction in APG TBL can be viewed as an effect of the balance between the increased turbulent-fluctuation and flow-deceleration contributions $c_{f}^{T}$ and $c_{f}^{A}$, respectively, and the decrease of the wall-normal convection contribution $c_{f}^{D 3}$.

A second implication of Eq. (13) is that the newly introduced acceleration term $c_{f}^{A}$ may be further decomposed, assuming that it is possible to distinguish between irrotational and rotational effects in the mean flow. In fact, we can formally write $c_{f}^{A}=c_{f}^{A B}+c_{f}^{A R}$, where $c_{f}^{A B}$ and $c_{f}^{A R}$ denote a contribution to the acceleration related to the dynamic pressure and a rotational one, respectively. If the Bernoulli equation is satisfied, it is possible to write

$$
c_{f}^{A}=c_{f}^{D 1}+c_{f}^{P}=-2 c_{f}^{P}+c_{f}^{P}=-c_{f}^{P},
$$


thus, we define the Bernoulli contribution $c_{f}^{A B}=-c_{f}^{P}$ to isolate the portion of $c_{f}^{A}$ that does not vanish if the Bernoulli equation holds. The rotational contribution to the acceleration term is then defined as the difference between the total acceleration term $c_{f}^{A}$ and the Bernoulli contribution, i.e., $c_{f}^{A R}=c_{f}^{A}-c_{f}^{A B}$. Note that $c_{f}^{A R}$ does not have an obvious analytical expression alternative to that obtained by substituting $c_{f}^{A}$ and $c_{f}^{A B}$ in its definition, i.e., $c_{f}^{A R}=\int_{0}^{1}\left(\xi_{f}^{D 1}+2 \xi_{f}^{P}\right) d \eta$. Introducing $c_{f}^{A R}$ and $c_{f}^{A B}$, the FIK decomposition can then be written as

$$
c_{f}=c_{f}^{T}+c_{f}^{A R}+c_{f}^{D 3}+c_{f}^{A B} .
$$

These four terms are shown in Fig. 12 (right). We observe that the Bernoulli-acceleration contribution $c_{f}^{A B}$ is always larger than the rotational contribution $c_{f}^{A R}$, while the latter is almost negligible in regions where the adverse pressure gradient is not very intense. In particular, at $x / c=0.6$ (where $\beta \approx 1.2), c_{f}^{A R}$ is less than one third of the turbulent-fluctuation contribution $c_{f}^{T}$, and it remains lower than $c_{f}^{T}$ up to $x / c=0.8$, where $\beta \approx 4.5$. The increasing values of $c_{f}^{A R}$ moving towards the trailing edge, where the flow is approaching near-separation conditions, can be seen as a consequence of the progressive distancing of the flow from a state well-described by the boundary-layer approximation. In fact, higher values of $c_{f}^{A R}$ are evidence of the diminishing influence of the free stream onto the turbulent region.

A more in-depth analysis of how the results of the FIK decomposition can be interpreted in case of strong pressure-gradient effects will require a systematic assessment of different data sets, including boundary layers under various pressure-gradient conditions and at various Reynolds numbers, and it is left for future studies. However, the results discussed in this section underline the complexity of friction generation for this type of flow, in particular when compared with cases with weak or zero streamwise inhomogeneity.

\section{Control effects}

Because of the streamwise development, the relative importance of the control effects on each term of the FIK decomposition is not uniform. In Fig. 13, we show the differences between controlled and uncontrolled cases at $\operatorname{Re}_{c}=200000$ for the seven contributions, denoted by $\Delta c_{f}^{\delta}$, $\Delta c_{f}^{T}, \Delta c_{f}^{D 1}, \Delta c_{f}^{D 2}, \Delta c_{f}^{D 3}, \Delta c_{f}^{D 4}$, and $\Delta c_{f}^{P}$, as well as the total $\Delta c_{f}^{D}$ and $\Delta c_{f}$ at $x / c=0.75$, which is the same location previously examined. At this streamwise location, the relative changes of $c_{f}$ are a reduction of almost $30 \%$ for uniform blowing and body-force damping on the suction side, an increase of similar amount for uniform suction on the suction side, and a reduction of $14 \%$ for blowing on the pressure side.

Because blowing increases both the ratio $\delta^{*} / \delta_{99}$ and the Reynolds number based on the displacement thickness, it decreases $c_{f}^{\delta}$, while suction increases it. However, these control effects are relatively small if compared to the total $c_{f}\left( \pm 2 \% c_{f}^{\text {ref. }}\right.$ ). Body-force damping, which has an even smaller impact on the boundary-layer thickness, leaves $c_{f}^{\delta}$ virtually unaltered $\left(+1 \% c_{f}^{\text {ref. }}\right)$.

The control effect on $c_{f}^{T}$ is a direct consequence of the alteration in $\overline{u_{t} v_{n}}$. In particular, uniform blowing on the suction side, which causes even stronger turbulent fluctuations in the outer layer, results in a higher $c_{f}^{T}$. Uniform suction, which causes lower turbulent fluctuations in the outer layer, results in a lower $c_{f}^{T}$. Uniform blowing over the pressure side also increases $c_{f}^{T}$, but by a lower amount than over the suction side. Note that blowing and suction, as well as the pressure gradient, affect $c_{f}^{T}$ in the opposite direction to their effects on the total $c_{f}$. Body-force damping, which reduces turbulent fluctuations to a level similar to that of uniform suction, causes a $c_{f}^{T}$ reduction. However, contrary to suction, such a reduction is one of the main effects of the control, being comparable with its global effect on $c_{f}$.

The control effects of blowing and suction on the streamwise-development and the pressuregradient terms are particularly interesting because they unveil qualitative differences between pressure gradient and control. Indeed, despite the fact that uniform-blowing effects on the turbulent 


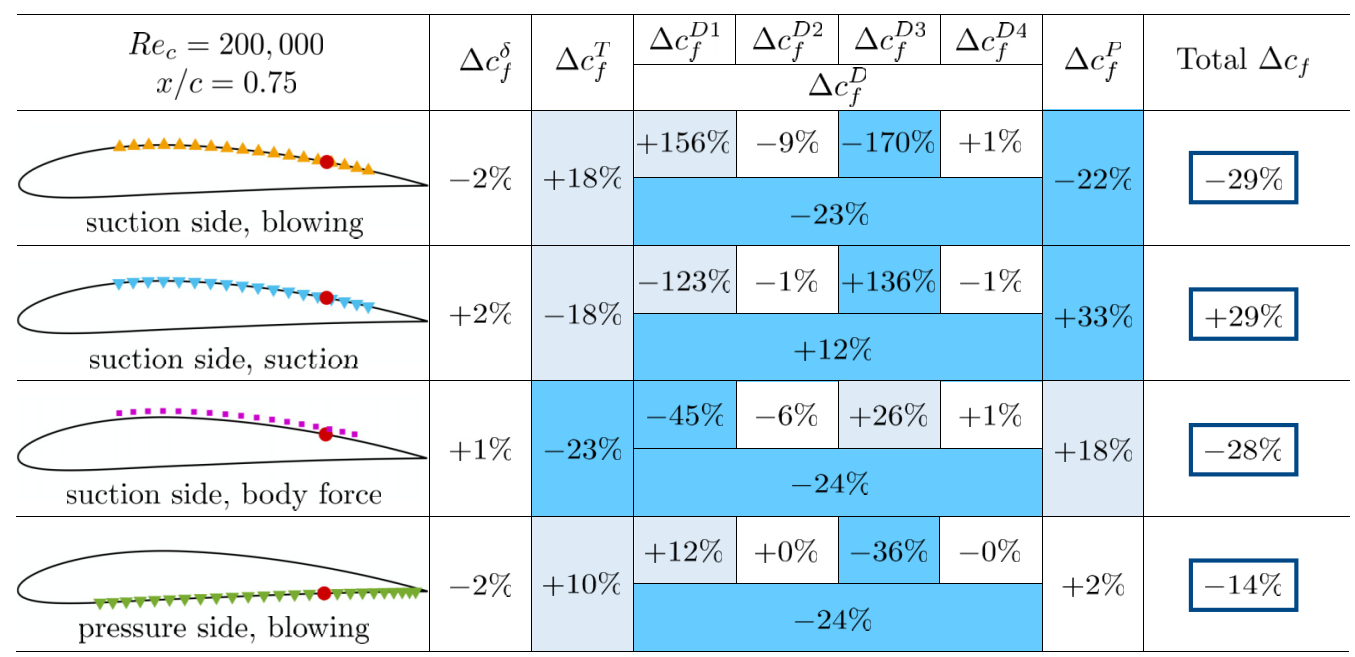

FIG. 13. Control effects on the terms of the FIK decomposition, normalized with the total $c_{f}$ of the reference case, e.g., $\Delta c_{f}^{\delta}=\left(c_{f}^{\delta}-c_{f}^{\delta \text {,ref. }}\right) / c_{f}^{\text {ref. }}$. By definition, $\Delta c_{f}^{D}=\Delta c_{f}^{D 1}+\Delta c_{f}^{D 2}+\Delta c_{f}^{D 3}+\Delta c_{f}^{D 4}$, and $\Delta c_{f}^{\delta}+$ $\Delta c_{f}^{T}+\Delta c_{f}^{D}+\Delta c_{f}^{P}=\Delta c_{f}$. Note that the differences are normalized with the reference $c_{f}$, so that the sum of all contributions gives the relative change of $c_{f}$. Colored cells denote the relevant changes in respect to the total $c_{f}$, and the darker color denotes the relevant changes which exhibit the dominant trend.

statistics share some similarities with those of a stronger adverse pressure gradient, $c_{f}^{D}$ is lower in cases with uniform blowing than in the reference. Furthermore, the small relative reduction of $c_{f}^{D}$ is high enough compared to the total $c_{f}$ to overcome the increase of the turbulent contribution $c_{f}^{T}$, which is part of the reason why uniform blowing results in lower $c_{f}$. A similar mechanism is observed on the pressure side, where uniform blowing results in a lower $c_{f}^{D}$, which is negative along most of the profile. Body-force damping also gives a slightly lower value of $c_{f}^{D}$. The control effects of blowing and suction on $c_{f}$ are significantly affected by the balance between contributions $c_{f}^{D 1}$ and $c_{f}^{D 3}$, and the latter, which is the term related to wall-normal convection, determines the increase or decrease of the skin friction for this kind of control. In particular, on both suction and pressure sides, the effects of uniform blowing are increasing $c_{f}^{D 1}$ and decreasing $c_{f}^{D 3}$, but uniform blowing decreases $c_{f}^{D 3}$, eventually resulting in skin-friction reduction. To the contrary, uniform suction increases $c_{f}^{D 3}$ and decreases $c_{f}^{D 1}$, but the increases of $c_{f}^{D 3}$ are higher than the decrease of $c_{f}^{D 1}$, so that uniform suction yields higher $c_{f}$. Body-force damping affects the development contribution differently than blowing and suction. In fact, its dominant effect is on the term related to the streamwise derivative $c_{f}^{D 1}$, which is reduced by a higher amount than the increase of $c_{f}^{D 3}$, resulting in lower $c_{f}^{D}$. Interestingly, the decrease of $c_{f}^{D}$ on the suction side due to the body force is comparable with that of $c_{f}^{T}$, an effect which cannot be observed when body-force damping is applied on zero-pressure-gradient boundary layers. We also observed that the control effects are negligible for $c_{f}^{D 4}$. Since this term is also always lower than $5 \%$ of the total $c_{f}$ for each case, it is probably reasonable to neglect it with only small errors if it is too difficult to estimate.

The pressure-gradient contribution $c_{f}^{P}$ is also affected by the control. On the suction side, uniform blowing reduces $c_{f}^{P}$ and uniform suction increases it. Body-force damping also has a relevant effect on $c_{f}^{P}$. Note that $c_{f}^{P}$ is negative in regions of strong APG, so that an increase in absolute value corresponds to a $c_{f}$ reduction, and vice versa. Thus, the $c_{f}^{P}$ reduction due to blowing and the $c_{f}^{P}$ increase due to suction and body-force damping indicate more and less intense pressure-gradient effects, respectively. Interestingly, the modifications of $c_{f}^{P}$ on the suction side are aligned with the 

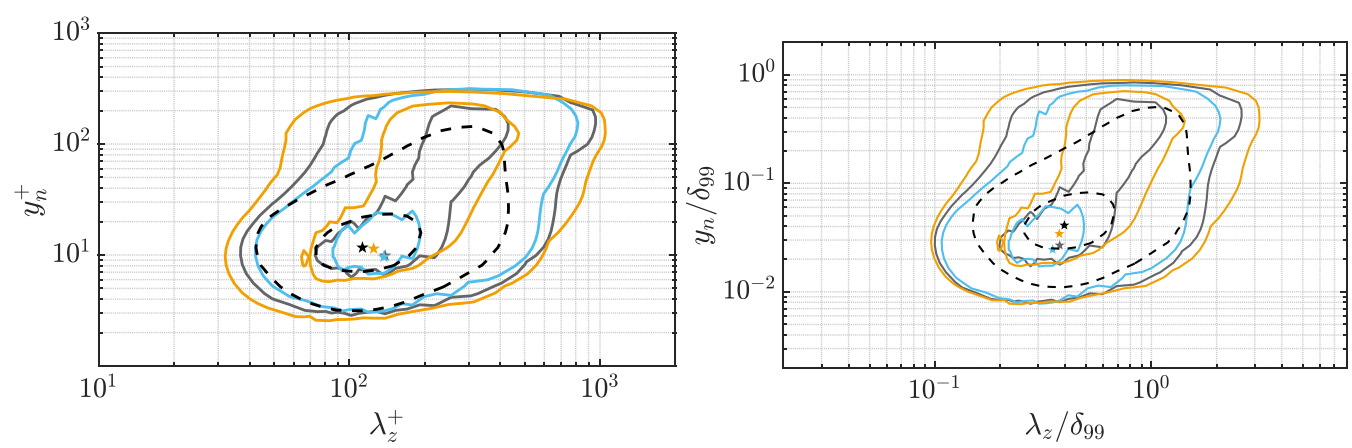

FIG. 14. Inner-scaled premultiplied spanwise power-spectral density of the wall-tangential Reynolds-shear stress for cases BL4 and SC4 and the uncontrolled suction side at $\operatorname{Re}_{c}=400000$, at location $x / c=0.75$, as functions of (left) the inner-scaled spanwise wavelength $\left(\lambda_{z}^{+}\right)$and wall-normal distance $\left(y_{n}^{+}\right)$, and (right) of the outer-scaled spanwise wavelength $\left(\lambda_{z} / \delta_{99}\right)$ and wall-normal distance $\left(y_{n} / \delta_{99}\right)$. The contours illustrate the levels corresponding to the $25 \%$ and the $75 \%$ of the maximum power density in the inner region for each case, and the locations of these maxima are marked with stars. Color code as in Table I, black lines for reference data of ZPG TBL at similar Reynolds number.

main control effect for blowing and suction ( $c_{f}$ reduction and increase, respectively), but opposite for body-force damping ( $c_{f}$ reduction). On the pressure side, uniform blowing leads to a slightly higher $c_{f}^{P}$, which is perhaps a consequence of the interaction between blowing and the weak but non-negligible favorable pressure gradient.

It is possible to observe that blowing and suction effects on the FIK contributions are not symmetric, in the sense that the decrease of $c_{f}^{D}$ due to uniform blowing is larger than the increase due to suction. At the same time, uniform suction increases $c_{f}^{P}$ more than uniform blowing decreases it. We examined the same contributions for the cases at $\operatorname{Re}_{c}=400000$, and we found that at higher Reynolds number the intensity of blowing and suction effects on the various terms are more similar. For instance, at $x / c=0,75, \Delta c_{f}^{D}=-15 \% c_{f}^{\text {ref }}$ and $\Delta c_{f}^{P}=-30 \% c_{f}^{\text {ref }}$ for uniform blowing, and $\Delta c_{f}^{D}=+16 \% c_{f}^{\mathrm{ref}}$ and $\Delta c_{f}^{P}=+36 \% c_{f}^{\mathrm{ref}}$ for uniform suction. This result may suggest that low-Reynolds-number effects are still present at $\operatorname{Re}_{c}=200000$. However, note that this is the sole qualitative difference between control cases at both $\operatorname{Re}_{c}$.

The control effects on the acceleration contribution introduced in the previous section, $c_{f}^{A}$, tend to follow those on the streamwise-development contribution $c_{f}^{D 1}$, so that $c_{f}^{A}$ is, e.g., increased by blowing and decreased by suction. The decomposition of the acceleration contribution into rotational acceleration $c_{f}^{A R}$ and acceleration related to the dynamic pressure $c_{f}^{A B}$ still does not allow to decouple the effect of the control because both $c_{f}^{A R}$ and $c_{f}^{A B}$ follow the total acceleration contribution $c_{f}^{A}$, i.e., they both increase or decrease by similar amounts when $c_{f}^{A}$ increases or decreases, respectively.

\section{SPECTRAL ANALYSIS}

In this section, we employ spectral analysis to investigate more in detail the scale-dependent interaction between adverse pressure gradient and uniform blowing and suction. We focus on the three cases at higher Reynolds number, and we also consider the turbulent boundary layer developing on the pressure side of the NACA4412 at $\operatorname{Re}_{c}=400000$ without control.

\section{A. One-dimensional spectra}

In Fig. 14 we illustrate contours of the inner-scaled premultiplied power-spectral density of the wall-tangential velocity fluctuations, denoted $k_{z} \phi_{u_{t}}^{+} u_{t}$, at $x / c=0.75$, expressed as a function of the 
spanwise wavelength $\lambda_{z}$ and the wall-normal distance $y_{n}$, scaled both in inner and outer units. The same quantities are also shown for a ZPG TBL at $\operatorname{Re}_{\tau} \approx 300$, for reference. Note that the spectra are computed using the fast Fourier transform (FFT) and that the contours in Fig. 14 represent $25 \%$ and $75 \%$ of the inner-peak power-spectral-density values of each case.

Tanarro et al. [41], who performed the same analysis on the uncontrolled case and on ZPG data at various Reynolds numbers, reported that the APG results in higher small-scale energy in the outer region, and higher large-scale energy in the near-wall region, in addition to the rise of the spectral outer peak. This is also apparent in our visualization. Blowing and suction effects on the powerspectral density of a ZPG boundary layer were studied by Kametani et al. [17], who found that blowing leads to higher values of the power-spectra density, with stronger effects in the outer region, while suction has opposite effects, and that blowing and suction do not alter the location of the spectral inner peak, although blowing leads to the rise of an outer peak. Similar observations can be made for the present data set, but the interactions with the adverse pressure gradient makes control effects even stronger. In the uncontrolled case, at the consider value of $x / c$, the inner and outer peaks have very similar spectral-density values 4.57 and 4.54, respectively. In case BL4, both values are higher than in the reference (5.65 and 7.24 for the inner and the outer peaks, respectively), but the outer peak becomes the location of highest power-spectral density. Furthermore, the inner-scaled wavelengths $\lambda_{z}^{+}$for both peaks are slightly lower in the case of blowing. This is perhaps due to the enhanced wall-normal convection, which not only leads to more energetic fluctuations at every wall-normal location, but also transports smaller structures far from the wall at a higher rate than in the reference. On the contrary, case SC4 exhibits lower power-spectral-density values for both inner and outer peaks (3.99 and 2.93, respectively), with the reduction being more prominent in relative terms for the outer peak. The wavelength of the inner peak in SC4 is in very good agreement with that of the reference, while that of the outer peak is slightly higher.

Interestingly, when the power-spectral densities are shown as functions of the outer-scaled wavelength and the wall-normal distance, the contours collapse for small scales close to the wall. However, in the same region, the distance between the APG data and the ZPG reference is higher in outer than in inner units. At the same time, the control effects on small-scale energy far from the wall and on the large-scale energy at every wall-normal distance are still evident. This fact suggests that uniform blowing and suction, at least at the moderate intensity considered here, affect mainly the properties of the large turbulent structures, and the transport of the small ones.

The control also affects the other power-spectral densities, although in a less pronounced way. The APG generally results in higher values of $k_{z} \phi_{v_{n} v_{n}}^{+}, k_{z} \phi_{w w}^{+}$, and $k_{z} \phi_{u_{t} u_{n}}^{+}$, in more energetic small scales in the near-wall region, and the convection of small-scale structures far from the wall. Uniform blowing amplifies those effects giving even higher spectral-density values, further energizing the small scale near the wall, and increasing wall-normal convection, and uniform suction has opposite effects. These observations are all in the direction of considering blowing and suction effects similar to those of pressure gradients.

\section{B. Two-dimensional spectra}

In Fig. 15 we illustrate contours of the two-dimensional power-spectral densities of the streamwise velocity fluctuations for cases BL4 and SC4 and the uncontrolled suction side at $\operatorname{Re}_{c}=400000$ at $x / c=0.75$, which is the same location examined above, and at two wall-normal distances, i.e., $y_{n}^{+}=15$ and 150 . Note that the power spectral density is computed via FFT for the periodic direction and using Welch's overlapping window method for time since the time series is not periodic.

Similarly to the one-dimensional power-spectral density, pressure-gradient and control effects modify both intensities and shapes of the two-dimensional spectra. In the uncontrolled case, at $y_{n}^{+}=$ 15 , the adverse pressure gradient results in higher spectral density values, e.g., the highest value is $\approx 2.0$ while the highest value at the same location of the pressure side is $\approx 1.7$. Furthermore, the period is reduced, and the contours expand in the direction of both shorter and longer wavelengths, for the smallest and largest structures, respectively. Uniform blowing (case BL4) amplified these 

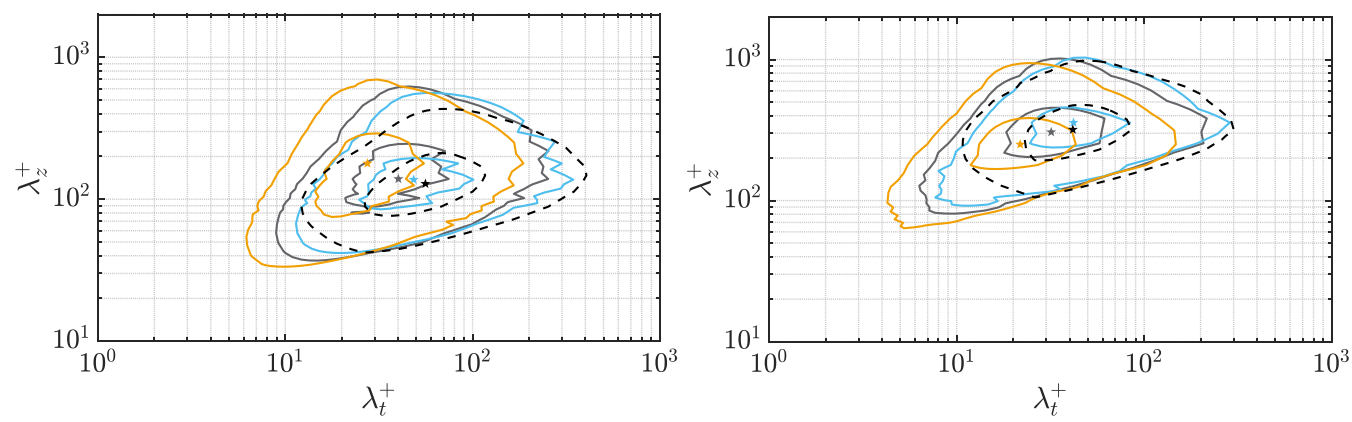

FIG. 15. Inner-scaled premultiplied spanwise and temporal power-spectral density of the wall-tangential velocity fluctuations at (left) $y_{n}^{+}=15$ and (right) $y_{n}^{+}=150$ for cases BL4 and SC4 and the uncontrolled suction side at $\operatorname{Re}_{c}=400000$, at location $x / c=0.75$. The contours illustrate the levels corresponding to the $25 \%$ and the $75 \%$ of the maximum power density for each case, and the locations of the maxima are marked with stars. Color code as in Table I, for reference data of ZPG TBL at similar Reynolds number.

effects: the highest power-spectral-density value is $\approx 2.4$, and it is located at an even higher $\lambda_{z}^{+}$ and a lower $\lambda_{t}^{+}$than in the reference. On the contrary, uniform suction (case SC4) at this location attenuates the effects of the adverse pressure gradient on the spectral density. In particular, the highest value is 1.6, and both the contours and the location of the maximum are in better agreement with those on the pressure side than with the uncontrolled case.

Farther from the wall, at $y_{n}^{+}=150$, adverse pressure gradient and uniform blowing lead to higher power-spectral densities while uniform suction to lower ones, but the locations of the maxima and the shapes of contours are modified in a different way compared with what we observe at $y_{n}^{+}=15$. The contours of all the cases are bounded by the line $\lambda_{z}^{+}=\alpha \lambda_{t}^{+(1 / 2)}$ on the lower-right end. Blowing and suction roughly shift the maxima along a line with the same slope, blowing towards shorter periods and wavelengths, and suction in the opposite direction. Interestingly, the location the highest power-spectral-density value for uniform suction has higher $\lambda_{z}^{+}$and $\lambda_{t}^{+}$not only with respect to the uncontrolled case, but also compared to the pressure side.

\section{DISCUSSION AND CONCLUSIONS}

In the previous sections, we analyzed the effects of uniform blowing, uniform suction, and body-force damping on the TBLs developing around a NACA4412 airfoil, in an effort to understand how these control strategies interact with the strong adverse pressure gradient in such flows. These results may help in future developments of control techniques applied to aerodynamic bodies. In particular, we discuss results of high-fidelity numerical simulations for uniform blowing and suction on the suction side of an airfoil at a chord Reynolds number up to $\operatorname{Re}_{c}=400000$. In many respects, uniform blowing and suction have effects qualitatively similar to those of APG and FPG, respectively. However, there are some notable exceptions for which this analogy does not hold. Furthermore, comparing blowing applied on ZPG and APG boundary layers reveals that, on the one hand, the skin-friction reduction or increase is similar in absolute terms but, on the other hand, the effects on the statistics and skin-friction contributions are amplified in the APG case.

The control effects on streamwise development are the consequence of changes in boundarylayer thickness and friction. For instance, blowing increases the boundary-layer thickness and decreases friction, resulting in higher $\beta$ and $\operatorname{Re}_{\theta}$, whereas suction decreases the boundary-layer thickness and increases friction, resulting in lower $\beta$ and $\operatorname{Re}_{\theta}$. In this case, blowing applied on the suction side has effects quite similar to those of an even more intense adverse pressure gradient. Blowing also results in a higher wall-tangential velocity in the wake region, in stronger wall-normal convection, in wall-tangential fluctuations with increased energy in the outer region and reduced 
energy in the inner region of the boundary layer, while uniform suction has opposite effects. Those are again qualitatively similar to pressure-gradient effects.

The most obvious difference between blowing and suction and pressure gradients is apparent in the inner-scaled profile of the wall-tangential velocity. In particular, while pressure gradients mainly affect the wake region of the boundary layer, blowing and suction modify the profiles at all wall-normal distances, as described by the Stevenson law [44]. Other interesting differences between control and pressure gradient arise examining more in detail their impact on the skin friction, which can be expressed in different contributions using the FIK indentity. Both APG and blowing result in lower skin friction, and eventually in separation, if they are intense enough. However, this is due to two different mechanisms. In an APG boundary layer, the contributions due to turbulent fluctuations and streamwise development are positive and progressively higher as $\beta$ increases but are counteracted by the pressure contribution, which is negative and rising in absolute value. Uniform blowing also increases the turbulent fluctuations, and further increases the absolute value of the negative pressure contribution, but it also decreases the streamwise-development term. These differences between pressure gradient and uniform blowing are even more evident comparing control effects on APG and ZPG boundary layers. On the pressure side, uniform blowing slightly increases the pressure contribution, which is positive, and eventually results in lower skin friction because of the reduction of the streamwise-development term. Furthermore, examining the FIK integrands unveils that the region where control effects are more intense moves farther from the wall when the control is applied to an APG boundary layer. Regarding the modification of the skin-friction contribution, suction has exactly the opposite effect to blowing, resulting in lower turbulent fluctuations but a higher streamwise-development term, and reducing the absolute value of the negative contribution.

Other insights into the impact of blowing and suction on APG TBLs are obtained through spectral analysis. In particular, the most evident effect is to energize or suppress, in the case of suction and blowing, respectively, the outer spectral peak of the wall-tangential velocity fluctuations. Furthermore, the locations of the maxima of power-spectral density in the outer region move towards shorter and shorter-lived structures for blowing, and towards larger and longer-lived structures for suction. This fact can potentially be due to the control effects on wall-normal convection, which enhances (in case of blowing) or reduces (in case of suction) the transfer of small structures away from the wall.

The observation that blowing and suction mainly affect the properties of large turbulent structures and the transport of small structures gives a hint to explain why the mean properties of APG TBL are more sensitive to these control strategies than the mean properties of ZPG TBL. The appearances of larger and more energetic coherent structures and more intense wall-normal transport are prominent pressure-gradient effects. Blowing and suction interact with these phenomena, so their impact on the flow is stronger when an adverse pressure gradient is present because the occurrence of intense events and wall-normal transport is more pronounced already in the uncontrolled case.

Body-force damping, although it has been calibrated to yield a skin-friction reduction similar to that of blowing, modifies the turbulent statistics in a qualitatively different way than blowing: it results in a higher mean wall-tangential velocity above the viscous sublayer, in wall-tangential fluctuations exhibiting higher energy in the inner region, and in less energetic structures in the outer region. When applied to an APG boundary layer, this type of control affects the turbulentfluctuation and the streamwise-development contributions with similar intensity, while it also has a non-negligible effect on the pressure-gradient term.

An important yet difficult point to discuss is how to generalize the present results, in particular related to the Reynolds number. Our investigation reaches $\operatorname{Re}_{c}=400000$, which corresponds to that of a glider airplane and it is not far from that of common academic experimental studies. Interestingly, for most local quantities, such as inner-scaled velocity profiles and skin friction, the comparison between cases with the same control at $\operatorname{Re}_{c}=200000$ and $\operatorname{Re}_{c}=400000$ shows negligible Reynolds-number effects. This result is possibly a consequence of the relatively similar values of the velocity actuation in inner units, and the choice of the present airfoil configuration that 
ensures a very similar pressure distribution at different Reynolds numbers. This may, however, be different case in more complex scenarios, e.g., different angles of attack, crossflow, and separation. Overall, the general question of how to design appropriate test cases for control strategies in external flows remains open and a worthwhile objective for future studies. The present results indicate, however, that the interaction between pressure gradient and actuation is nontrivial, and thus the effects of both cannot be easily separated. We can therefore conclude that further analysis on this topic would benefit from using complex flow cases, in particular, including varying flow conditions such as pressure gradients.

A second interesting point left for future studies is the identification of the most convenient framework to analyze friction generation in case of strong pressure-gradient effects or other sources of streamwise inhomogeneity. Both the identities proposed by Fukagata et al. [18] and Renard and Deck [43] tend to give skin-friction contributions with very high values in these cases. The total $c_{f}$ is then recovered as a result of multiple cancellations, which may be difficult to explain from a physical perspective. We propose an alternative form of aggregating the FIK contributions, based on the connection between the term related to streamwise development of the mean wall-tangential velocity component and the dynamic pressure. However, future efforts will be needed to clarify the interpretation of the friction decomposition in nonhomogeneous wall-bounded turbulent flows.

As a final remark we should state that we did not investigate the possible impact of using a simple Dirichlet boundary condition to represent the control actuation. Employing a more involved model for MBT surfaces (or similar implementations) would allow both a more realistic description of the flow as well as an estimate of the actuation cost. The latter point is of course of relevance from the control-effectiveness perspective, which is, however, separate from the fluid-dynamics considerations presented in this paper.

The data of this paper will be made available online [45].

\section{ACKNOWLEDGMENTS}

This study was funded by the Swedish Foundation for Strategic Research, project "In-Situ Big Data Analysis for Flow and Climate Simulations" (Project No. BD15-0082), by the Knut and Alice Wallenberg Foundation and by the Swedish Research Council (VR). D.G. and B.F. acknowledge partial support through DFG Priority Programme 1881 "Turbulent Superstructures." The simulations were performed on resources provided by the Swedish National Infrastructure for Computing (SNIC) and within the project CWING on the national supercomputer Cray XC40 Hazel Hen at the High Performance Computing Center Stuttgart (HLRS). We acknowledge an anonymous reviewer for inspiration on the formulation of alternative aggregations of the FIK contributions described in Sec. V B.

\section{APPENDIX A: FIT TO NICKELS FORMULA}

The formula proposed by Nickels [40] has been employed in previous experimental studies to estimate the local skin-friction coefficient from velocity measurement (e.g., [11]). This formula consists of a composite function for $U^{+}$with three different terms, denoted $U^{+}\left(y^{+}, \eta\right)=f\left(y^{+}\right)+$ $g\left(y^{+}, \eta\right)+w\left(y^{+}, \eta\right)$ hereafter. The first term describes the profile in the near-wall region, and it reads as

$$
f\left(y^{+}\right)=y_{c}^{+}\left[1-\left(1+2\left(\frac{y^{+}}{y_{c}^{+}}\right)+\frac{1}{2}\left(3-p_{x}^{+} y_{c}^{+}\right)\left(\frac{y^{+}}{y_{c}^{+}}\right)^{2}-\frac{3}{2} p_{x}^{+} y_{c}^{+}\left(\frac{y^{+}}{y_{c}^{+}}\right)^{3}\right) e^{-3 y^{+} / y_{c}^{+}}\right] .
$$

The second term describes the logarithmic region of the profile, and it reads as

$$
g\left(y^{+}, \eta\right)=\frac{\sqrt{1+p_{x}^{+} y_{c}^{+}}}{6 k_{0}} \ln \left(\frac{1+\left[0.6\left(y^{+} / y_{c}^{+}\right)\right]^{6}}{1+\eta^{6}}\right) \text {. }
$$



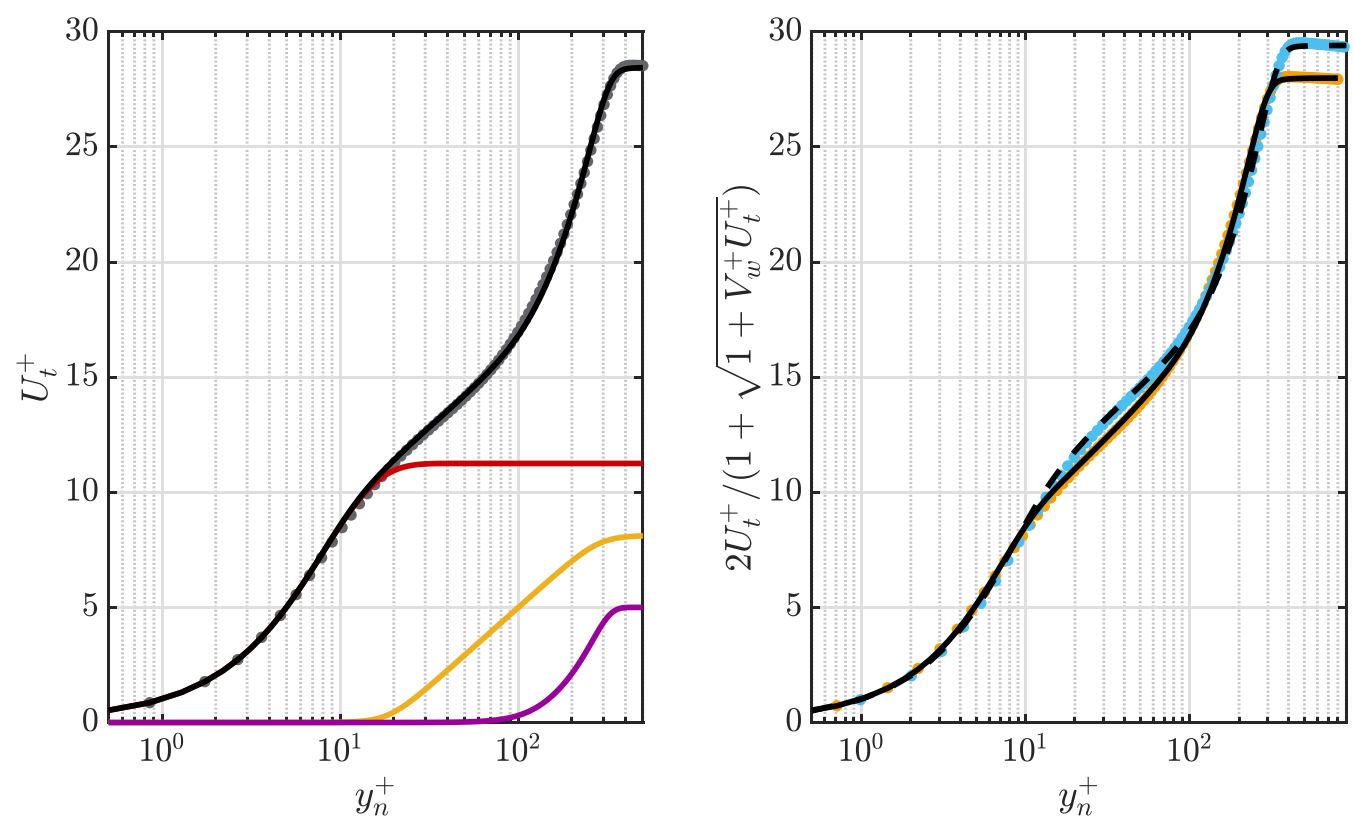

FIG. 16. Profiles from Nickels formula [40] for (left) the inner-scaled wall-tangential velocity of the reference case at $R e_{c}=400,000$ and streamwise location $x / c=0.75$ and (right) the wall-tangential velocity scaled with the Stevenson law for blowing and suction at same $R e_{c}$ and $x / c$. The color code for the velocity profiles is the same as in in Table I. The black lines denote the profiles from Nickels fit (solid and dashed line on the left plot denote blowing and suction, respectively). The red, orange, and purple lines denote the linear term (A1), the logarithmic term (A2), and the wake term (A3) for the reference case, respectively.

The third term is related to the wake, and it is written as

$$
w(\eta)=b\left(1-e^{\frac{5\left(\eta^{4}+\eta^{8}\right)}{\left(1+5 \eta^{3}\right)}}\right)
$$

In these expressions, $y^{+}$and $\eta=y_{n} / \delta$ denote the inner-scaled wall-normal distance (we drop the subscript $n$ in $y^{+}$for simplicity), and the wall-normal distance scaled with an estimated boundary-layer thickness $\delta$; the constant term $y_{c}^{+}$is an estimate for the critical location at which the viscous sublayer becomes unstable; $p_{x}^{+}=\left(v / \rho u_{\tau}^{3}\right)(d p / d x)$ is the normalized pressure gradient in the proximity of the wall; $b$ measures the wake strength; and $k_{0}=0.39$ is a modified von Kármán constant.

Since our aim is to determine whether this theoretical formula describes our data, rather than if it can be used to extract information from measurements, we performed the fit in inner units, defining $\eta=y^{+} l^{*} / \delta$ (where $l^{*}=v / u_{\tau}$ ). We fixed the value of $p_{x}^{+}$, using the pressure gradient at the wall and we fit our data letting $y_{c}^{+}, \delta$, and $b$ vary. Note that the value of $y_{c}^{+}$can be computed from the data if the value of the critical Reynolds number of the sublayer, denoted by $R_{c}=u_{\tau} y_{c} / \nu$, is known. However, the value of $R_{c}$ needs to be estimated, for instance from ZPG data, and $p_{x}^{+}$also enters the formula to compute $y_{c}^{+}$. We obtain virtually the same agreement with the data fixing $p_{x}^{+}$or letting it vary, obtaining different values of $y_{c}^{+}$(which correspond to different values of $R_{c}$ ). A worse agreement is inevitable if both $p_{x}^{+}$and $y_{c}^{+}$are fixed. We did not find a convincing way to determine whether it is more desirable to fix $p_{x}^{+}$or $R_{c}$. We did not let $k_{0}$ vary, which would allow to improve the agreement further, but it has no justification given the virtually absent logarithmic region in this data set. 

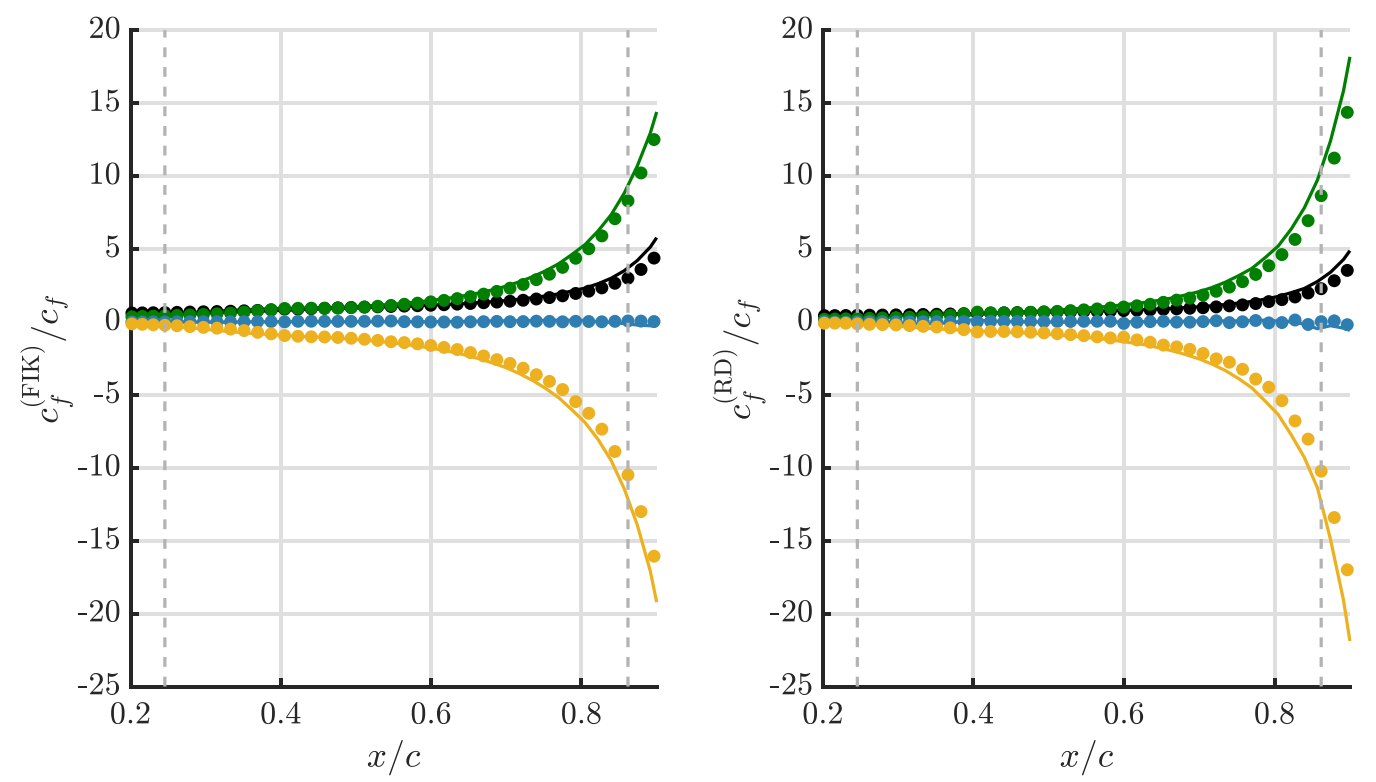

FIG. 17. Comparison between relative contributions to the local skin-friction coefficient $c_{f}$ over the airfoil suction side, for the reference cases at (lines) $\operatorname{Re}_{c}=200000$ and (symbols) $\operatorname{Re}_{c}=400000$, computed using the (left) FIK and (right) RD decomposition. Contributions derived from similar terms of the governing equations are grouped together: $(\square)$ Reynolds-shear stress $\left(c_{f}^{T}\right.$ and $\left.c_{f 2}^{\mathrm{RD}}\right),(\square)$ mean convection $\left(c_{f}^{D 1}+c_{f}^{D 3}\right.$ and $\left.c_{f 31}^{\mathrm{RD}}\right),($ streamwise derivatives $\left(c_{f}^{D 2}+c_{f}^{D 4}\right.$ and $\left.c_{f 32}^{\mathrm{RD}}\right)$, and ( $\square$ ) pressure gradients $\left(c_{f}^{P}\right.$ and $\left.c_{f 33}^{\mathrm{RD}}\right)$.

The results of the fit are shown in Fig. 16 for streamwise location $x / c=0.75$ for the reference case at $\operatorname{Re}_{c}=400000$, and the profiles scaled with the Stevenson law for blowing and suction at the same Reynolds number, with a relatively good agreement in all cases.

At this location, for the reference case, $p_{c}^{+}$is $\approx 0.03$ based on the edge location estimated with the diagnostic plot, for which $y_{c}^{+} \approx 11.2$ is found from the fit. If the pressure gradient is not fixed, the fit estimates $p_{c}^{+} \approx 0.05$ and $y_{c}^{+} \approx 11.9$.

Note that the range for which the formula has been tested by Nickels is $-0.02<p_{x}^{+}<0.06$, but the low $\operatorname{Re}_{\tau}$ of the current data set makes the fitting procedure more difficult for logarithmic function $g\left(y^{+}, \eta\right)$. A similar agreement with the data was obtained even for streamwise location $x / c=0.8$, where $p_{c}^{+} \approx 0.05$, and a better agreement was obtained at lower $x / c$, where the pressure-gradient effects are less intense (not shown here).

\section{APPENDIX B: COMPARISON BETWEEN FIK AND RD DECOMPOSITIONS}

The decomposition derived by Renard and Deck [43], denoted by RD hereafter, has been developed specifically to overcome the limitations of the FIK identity, namely, the fact that the triple integration by parts in the derivation of the latter does not have a clear physical interpretation, and the resulting integrands do not have intuitive scaling properties. The RD identity is obtained in a frame of reference where the wall is moving with the free-stream velocity, and the upper limit of the integration is the free stream. The best generalization of this expression in the case of the boundary layer on an airfoil is not obvious, but a possible solution is to assume $U_{\infty}=U_{e}$ and $\delta_{99}$ as upper limit. Although this assumption is against the spirit of the RD decomposition, we verified that extending the integration farther from the wall does not have a significant impact for the qualitative discussion presented here. In the RD decomposition, the skin friction is expressed as a sum of three contributions: $c_{f}=c_{f 1}^{\mathrm{RD}}+c_{f 2}^{\mathrm{RD}}+c_{f 3}^{\mathrm{RD}}$. The first term is related to viscous dissipation, the second 
term is related to TKE production, and the third term is related to the boundary-layer development:

$$
c_{f 1}^{\mathrm{RD}}=\frac{2}{U_{e}^{3}} \int_{0}^{\delta_{99}} v\left(\frac{\partial U_{t}}{\partial y_{n}}\right)^{2} d y_{n}, \quad c_{f 2}^{\mathrm{RD}}=\frac{2}{U_{e}^{3}} \int_{0}^{\delta_{99}}-\overline{u v} \frac{\partial U}{\partial y} d y, \quad c_{f 3}^{\mathrm{RD}}=\frac{2}{U_{e}^{3}} \int_{0}^{\delta_{99}}\left(U-U_{e}\right) \frac{\partial \tau}{\partial y} d y .
$$

This last term can be also expressed as the sum of three contributions [43], denoted by $c_{f 3}^{\mathrm{RD}}=$ $c_{f 31}^{\mathrm{RD}}+c_{f 32}^{\mathrm{RD}}+c_{f 33}^{\mathrm{RD}}$ :

$$
\begin{aligned}
& c_{f 31}^{\mathrm{RD}}=\frac{2}{U_{e}^{3}} \int_{0}^{\delta_{99}}\left(U-U_{e}\right)\left(U \frac{\partial U}{\partial x}+V \frac{\partial U}{\partial y}\right) d y, \quad c_{f 32}^{\mathrm{RD}}=\frac{2}{U_{e}^{3}} \int_{0}^{\delta_{99}}-\left(U-U_{e}\right)\left(\nu \frac{\partial^{2} U}{\partial x^{2}}-\frac{\partial \overline{u u}}{\partial x}\right) d y, \\
& c_{f 33}^{\mathrm{RD}}=\frac{2}{U_{e}^{3}} \int_{0}^{\delta_{99}}-\left(U-U_{e}\right)\left(\frac{1}{\rho} \frac{\partial p}{\partial x}\right) d y .
\end{aligned}
$$

The RD contributions cannot be directly written in terms of the FIK identity, but it still is possible to verify whether the two decompositions lead to qualitatively different results by regrouping contributions that derive from the same terms of the governing equations. In particular, $c_{f}^{T}$ and $c_{f 2}^{\mathrm{RD}}$ are both related to the Reynolds shear stress, $c_{f}^{D 1}+c_{f}^{D 3}$ and $c_{f 31}^{\mathrm{RD}}$ are both related to mean convection, $c_{f}^{D 2}+c_{f}^{D 4}$ and $c_{f 32}^{\mathrm{RD}}$ are both related to streamwise derivatives, and $c_{f}^{P}$ and $c_{f 33}^{\mathrm{RD}}$ are both related to the pressure gradient. The relative proportion of these terms with respect to the total $c_{f}$ is illustrated in Fig. 17 for the suction side of the reference cases at both $\operatorname{Re}_{c}=200000$ and $\operatorname{Re}_{c}=400000$. Although the corresponding contributions do not have the same numerical values, they exhibit a remarkably similar behavior. We observed that the same results also hold for the cases with control (not shown here), showing that our conclusions do not depend on the specific skin-friction decomposition chosen for the analysis.

[1] L. Prandtl, Über Flüssigkeitsbewegung bei sehr kleiner Reibung, Proc. Third Int. Math. Cong. 2, 484 (1904).

[2] L. Prandtl and A. Betz, Ergebnisse der Aerodynamischen Versuchsanstalt zu Göttingen. IV. Lieferung (Göttingen University Press, Göttingen, 1932).

[3] P. Duwez and H. L. J. Wheeler, Experimental study of cooling by injection of a fluid through a porous material, J. Aeronaut. Sci. 15, 509 (1948).

[4] T. Black and A. Sarnecki, The Turbulent Boundary Layer with Suction or Injection (Ministry of Aviation, London, 1965).

[5] H. Julien, W. Kays, and R. Moffat, The Turbulent Boundary Layer on a Porous Plate: An Experimental Study of the Effects of a Favorable Pressure Gradient (Ministry of Aviation, London, 1965).

[6] R. Baker, The Transpired Turbulent Boundary Layer with Streamwise Pressure Gradient (Imperial College Press, London, 1971).

[7] D. Hwang, A Proof of Concept Experimentfor Reducing Skin Friction by Using a Micro-blowing Technique, NASA, TM (AIAA, Reston, VA, 1996).

[8] D. Hwang, Review of research into the concept of the microblowing technique for turbulent skin friction reduction, Prog. Aerosp. Sci. 40, 559 (2004).

[9] V. Kornilov, Current state and prospects of researches on the control of turbulent boundary layer by air blowing, Prog. Aerosp. Sci. 76, 1 (2015).

[10] V. Kornilov, Implementation of air injection into the turbulent boundary layer of aircraft wing using external pressurized flow, Thermophys. Aeromech. 24, 175 (2017).

[11] K. Eto, Y. Kondo, K. Fukagata, and N. Tokugawa, Assessment of friction drag reduction on a Clark-Y airfoil by uniform blowing, AIAA J. 57, 2774 (2019). 
[12] V. Kornilov, I. Kavun, and A. Popkov, Modification of turbulent airfoil section flow using a combined control action, Thermophys. Aeromech. 26, 165 (2019).

[13] V. I. Kornilov, Combined blowing/suction flow control on low-speed airfoils, Flow Turbul. Combust. 106, 81 (2021).

[14] H. Choi, P. Moin, and J. Kim, Active turbulence control for drag reduction in wall-bounded flows, J. Fluid Mech. 262, 75 (1994).

[15] Y. Sumitani and N. Kasagi, Direct numerical simulation of turbulent transport with uniform wall injection and suction, AIAA J. 32, 1220 (1995).

[16] Y. Kametani and K. Fukagata, Direct numerical simulation of spatially developing turbulent boundary layers with uniform blowing or suction, J. Fluid Mech. 681, 154 (2011).

[17] Y. Kametani, K. Fukagata, R. Örlü, and P. Schlatter, Effect of uniform blowing/suction in a turbulent boundary layer at moderate Reynolds number, Int. J. Heat Fluid Flow 55, 134 (2015).

[18] K. Fukagata, K. Iwamoto, and K. N., Contribution of reynolds stress distribution to the skin friction in wall-bounded flows, Phys. Fluids 14, L73 (2002).

[19] A. Stroh, B. Frohnapfel, P. Schlatter, and Y. Hasegawa, A comparison of opposition control in turbulent boundary layer and turbulent channel flow, Phys. Fluids 27, 075101 (2015).

[20] A. Stroh, Y. Hasegawa, P. Schlatter, and B. Frohnapfel, Global effect of local skin friction drag reduction in spatially developing turbulent boundary layer, J. Fluid Mech. 805, 303 (2016).

[21] C. Wenzel and U. Rist, Application of the von Kármán momentum theorem to turbulent boundary layers with uniform blowing, IAG Institutsbericht, IB16-01 (Universität Stuttgart, Stuttgart, 2016).

[22] M. Ferro, B. E. Fallenius, and J. H. Fransson, Experimental study on turbulent asymptotic suction boundary layers, J. Fluid Mech. 915, A80 (2021).

[23] A. Bobke, R. Örlü, and P. Schlatter, Simulations of turbulent asymptotic suction boundary layers, J. Turbul. 17, 157 (2016).

[24] O. A. Mahfoze, A. Moody, A. Wynn, R. D. Whalley, and S. Laizet, Reducing the skin-friction drag of a turbulent boundary-layer flow with low-amplitude wall-normal blowing within a bayesian optimization framework, Phys. Rev. Fluids 4, 094601 (2019).

[25] M. Albers, P. Meysonnat, and W. Schröder, Actively reduced airfoil drag by transversal surface waves, Flow Turbul. Combust. 102, 865 (2019).

[26] R. Vinuesa and P. Schlatter, Skin-friction control of the flow around a wing section through uniform blowing, in 16th European Turbulence Conference (ETC), 21-24 August, Stockholm, 2017 (unpublished).

[27] M. Atzori, R. Vinuesa, G. Fahland, A. Stroh, D. Gatti, B. Frohnapfel, and P. Schlatter, Aerodynamic Effects of Uniform Blowing and Suction on a NACA4412 Airfoil, Flow Turbul. Combust. 105, 735 (2020).

[28] G. Fahland, A. Stroh, B. Frohnapfel, M. Atzori, R. Vinuesa, P. Schlatter, and D. Gatti, Investigation of blowing and suction for turbulent flow control on airfoils, AIAA J. 59, 4422 (2021).

[29] P. Fischer, J. Lottes, and S. Kerkemeier, Nek5000: Open source spectral element CFD solver, available at http://nek5000.mcs.anl.gov.

[30] P. Schlatter, S. Stolz, and L. Kleiser, LES of transitional flows using the approximate deconvolution model, Int. J. Heat Fluid Flow 25, 549 (2004).

[31] R. Vinuesa, P. Negi, M. Atzori, A. Hanifi, D. Henningson, and P. Schlatter, Turbulent boundary layers around wing sections up to $R e_{c}=1,000,000$, Int. J. Heat Fluid Flow 72, 86 (2018).

[32] P. Schlatter and R. Örlü, Assessment of direct numerical simulation data of turbulent boundary layers, J. Fluid Mech. 659, 116 (2010).

[33] R. Vinuesa, A. Bobke, R. Örlü, and P. Schlatter, On determining characteristic length scales in pressuregradient turbulent boundary layers, Phys. Fluids 28, 055101 (2016).

[34] P. H. Alfredsson, A. Segalini, and R. Örlü, A new scaling for the streamwise turbulence intensity in wall-bounded turbulent flows and what it tells us about the 'outer' peak, Phys. Fluids 23, 041702 (2011).

[35] S. Dong, G. E. Karniadakis, and C. Chryssostomidis, A robust and accurate outflow boundary condition for incompressible flow simulations on severely-truncated unbounded domains, J. Comput. Phys. 261, 83 (2014). 
[36] P. Schlatter and R. Örlü, Turbulent boundary layers at moderate Reynolds numbers: Inflow length and tripping effects, J. Fluid Mech. 710, 5 (2012).

[37] S. M. Hosseini, R. Vinuesa, P. Schlatter, A. Hanifi, and D. S. Henningson, Direct numerical simulation of the flow around a wing section at moderate Reynolds number, Int. J. Heat Fluid Flow 61, 117 (2016).

[38] G. Eitel-Amor, R. Örlü, and P. Schlatter, Simulation and validation of a spatially evolving turbulent boundary layer up to $R e_{\theta}=8300$, Int. J. Heat Fluid Flow 47, 57 (2014).

[39] T. N. Stevenson, A law of the wall for turbulent boundary layers with suction or injection., CoA Report Aero. No. 166, 1963 (unpublished).

[40] T. B. Nickels, Inner scaling for wall-bounded flows subject to large pressure gradients, J. Fluid Mech. 521, 217 (2004).

[41] A. Tanarro, R. Vinuesa, and P. Schlatter, Effect of adverse pressure gradients on turbulent wing boundary layers, J. Fluid Mech. 883, A8 (2020).

[42] M. Yoon, J. Ahn, J. Hwang, and H. J. Sung, Contribution of velocity-vorticity correlations to the frictional drag in wall-bounded turbulent flows, Phys. Fluids 28, 081702 (2016).

[43] N. Renard and S. Deck, A theoretical decomposition of mean skin friction generation into physical phenomena across the boundary layer, J. Fluid Mech. 790, 339 (2016).

[44] I. Vigdorovich, A law of the wall for turbulent boundary layers with suction: Stevenson's formula revisited., Phys. Fluids 28, 085102 (2016).

[45] See, https://www.flow.kth.se/flow-database. 